\title{
Robust inventory control under demand and lead time uncertainty
}

\section{Authors: Andreas Thorsen \& Tao Yao}

The final publication is available at Springer via http://dx.doi.org/10.1007/s10479-015-2084-1.

Thorsen, Andreas, and Tao Yao. "Robust inventory control under demand and lead time uncertainty." Annals of Operations Research (December 2015): 1-30.

DOI: $10.1007 / \mathrm{s} 10479-015-2084-1$

Made available through Montana State University's $\underline{\text { ScholarWorks }}$

scholarworks.montana.edu 


\title{
Robust Inventory Control Under Demand and Lead Time Uncertainty
}

\author{
Andreas Thorsen - Tao Yao
}

Received: date / Accepted: date

\begin{abstract}
In this paper a general methodology is proposed based on robust optimization for an inventory control problem subject to uncertain demands and uncertain lead times. Several lead time uncertainty sets are proposed based on the budget uncertainty set, and a set based on the central limit theorem (CLT). Robust optimization models are developed for a periodic review, finite horizon inventory control problem subject to uncertain demands and uncertain lead times. We develop an approach based on Benders' decomposition to compute optimal robust (i.e., best worst-case) policy parameters. The proposed approach does not assume distributional knowledge, makes no assumption regarding order crossovers, and is tractable in a practical sense. Comparing the new approach to an epigraph reformulation method, we demonstrate that the epigraph reformulation approach is overly conservative even when costs are stationary. The approach is benchmarked against the sample average approximation (SAA) method. Computational results indicate that the approach provides more stable and robust solutions compared to SAA in terms of standard deviation and worst-case solution, especially when the realized distribution is different than the sampled distribution.
\end{abstract}

Keywords robust optimization · inventory control · lead time uncertainty · demand uncertainty

\section{Introduction}

In supply chain systems where supply and demand are uncertain it is important to calculate optimal inventory ordering policies to reduce costs while maintaining high customer service levels. Future demand may be difficult to predict and lead

\author{
A. Thorsen \\ Jake Jabs College of Business \& Entrepreneurship, Montana State University, 302 Jabs Hall, \\ Bozeman MT 59717, USA \\ E-mail: andreas.thorsen@montana.edu \\ T. Yao \\ Harold and Inge Marcus Department of Industrial and Manufacturing Engineering, Pennsyl- \\ vania State University, 310 Leonhard Building, University Park, PA 16801, USA
}


times may be uncertain because of reasons such as variable processing times at the supplier or transportation delays. There is a rich body of literature dealing with inventory control under demand uncertainty, and there is a much smaller body of literature concerning inventory control under lead time uncertainty.

Formulating an inventory control problem under lead time uncertainty is difficult because of a phenomenon called "order crossover", which is the arrival of orders in a sequence different than the sequence they were placed (He et al. 1998). Order crossover distorts the lead time distribution which complicates the analysis.

Many results in the literature rely on the assumption that order crossovers do not occur and that orders are independent and identically distributed (i.i.d) but the i.i.d. assumption is contradictory and this assumption will not always hold (Hayya et al. 2008). In fact, crossovers are likely to occur more frequently in the future for several reasons. Riezebos (2006) examines the changes in modern supply chain management and concludes that some of the reasons for the increasing frequency of order crossovers are "a reduction of the time between issuing an order, more suppliers, more frequent ordering, larger distances between supplier and firm, more supply options with different lead time consequences, and dependable but more variable total lead times."

Many policies have been proposed for inventory problems under stochastic demand and constant lead time. For example, the basestock policy (also called the "order-up-to" policy), which involves placing an order for $S-x$ units when the inventory position $x$ falls below $S$, was initially proven to be optimal for serial supply chains when demand follows a known distribution by Clark and Scarf (1960) and it has been shown to be optimal for more general systems since then (the reader is referred to Zipkin (2000) where many extensions can be found). Due to its simplicity, the basestock policy has been widely adopted in industry. Stochastic dynamic programming (SDP) is often used to compute the optimal policy parameters. However, SDP is intractable for large problems as it suffers from "the curse of dimensionality" (Zipkin 2000). Another downside of SDP is that for many real world problems the true probability distribution may not be known.

An alternate approach to handle uncertainty is using uncertainty sets in a robust optimization (RO) framework. RO has been studied in supply chain problems showing promising computational results for problems under demand uncertainty (e.g., see Ben-Tal et al. 2005, Bertsimas and Thiele 2006, Bienstock and Ozbay 2008). In these papers robust optimization is cast as a distribution-free approach that does not take into account information about probability distributions or available data on past parameter realizations. In the above papers involving demand uncertainty, the supply-side is assumed to be deterministic and order lead times are assumed to be either zero or fixed. There have been far fewer RO papers on supply uncertainty, and they mainly deal with yield uncertainty and raw material supply uncertainty. These papers are discussed in Section 2.

In this paper a general methodology is proposed based on robust optimization for an inventory control problem subject to uncertain lead times as well as uncertain demand. Prior research has examined this problem using stochastic dynamic programming under the assumption of no order crossovers and full distributional knowledge of lead times. Important characteristics of the proposed approach is that it does not assume distributional knowledge, it makes no assumption regard- 
ing order crossovers, and it is tractable in a practical sense. The contributions of this paper are as follows:

- We propose a RO model for an inventory control problem under uncertain demand and lead time. While there are several results in the literature for robust inventory control under uncertain demand, this is the first that jointly considers uncertain demand and uncertain lead time from an RO perspective. We present a new budget-type lead time uncertainty set as well as a CLT-based lead time uncertainty set.

- We use a Benders' decomposition approach to handle robust optimization problems under CLT-based uncertainty sets. The Benders' approach has been shown to produce less conservative solutions than the alternative method in the literature of robustifying the epigraph formulation of inventory control problems (Gorissen and Den Hertog 2013).

- Using a computational study for an inventory control problem we compare the performance of several uncertainty set modeling approaches (budget and CLT). This is the first attempt to compare the performance of these uncertainty sets in this setting. The numerical results indicate that our approach compares well to Sample Average Approximation (SAA).

In Section 2 we discuss relevant literature. In Section 3 we describe the RO methodology including several modeling approaches for uncertainty sets. We present the robust inventory problem in Section 4. The solution approach is described in Section 5. In Section 6, we extend the approach to involve more general lead time uncertainty sets. Computational results follow in Section 7. Finally, concluding remarks are made in Section 8.

\section{Literature Review}

\subsection{Robust Optimization}

Robust optimization (RO) is a methodology used to solve optimization problems that involve uncertainty in the parameter values without considering probability distributions. Typically, the objective of robust optimization is to find the best worst-case solution over an uncertainty set specified by the modeler. The set-based robust optimization is significantly different than the scenario-based robust optimization developed in Mulvey et al. (1995). In scenario-based robust optimization a finite set of scenarios are considered and the solution may violate the constraints involving these scenarios; a penalty function is included in the objective to account for the violations. For the remainder of the paper, when we write RO we are referring to set-based robust optimization.

The downside of the RO approach is that the solutions are usually more conservative than solutions generated using complementary methods such as stochastic optimization, although the conservatism can be controlled somewhat by the design of the uncertainty sets (Ben-Tal et al. 2009). The main advantage of robust optimization over alternative methods (e.g., stochastic dynamic programming) is that the approach is computationally tractable (i.e., polynomially solvable) for many cases. For example, a robust linear program with an ellipsoidal uncertainty set can be reformulated as a second-order cone problem which is tractably solvable using 
interior-point methods (Ben-Tal and Nemirovski 1999), and a robust linear program with polyhedral uncertainty can be reformulated as a (slightly larger) linear program (Bertsimas and Sim 2004). However, these reformulations are possible because of an approach using duality to handle subproblems involving uncertain parameters in the constraints of the optimization problems. Therefore, uncertainty sets which are row-wise dependent are considered. We refer the reader to Gabrel et al. (2014), a recent survey paper on applications of robust optimization, which describes this reformulation approach. When uncertainty is dependent within the columns (as is the case for the lead time models presented in this paper), this reformulation tactic does not work (see section Differences with Bertsimas and Sim's Approach in Minoux (2009)).

Widely cited as the first paper considering RO, Soyster (1973) considers a model using column-wise uncertainty sets for linear programming problems where the uncertainty sets are ellipsoids. The author shows that if each column of the constraint matrix belongs to a convex set, then solving the problem amounts to solving a linear program with the matrix coefficients equal to their worst-case value. While this is beautifully simple, it is also an extremely conservative modeling approach. The formulation by Soyster was largely dismissed for many years because of its extreme conservatism. Ben-Tal and Nemirovski (1999) formulate a robust optimization model for a linear program where the uncertainty is rowwise and ellipsoidal. They show that their model can be reformulated as a conic quadratic program. Bertsimas and Sim (2004) formulate an alternative robust optimization model using a row-wise and polyhedral uncertainty set in which a budget parameter is used to control the conservatism of the model. The main advantage of this method is that the robust counterpart of a linear program under budget uncertainty remains a (slightly larger) linear program, while the robust counterpart of a linear program under the Ben-Tal and Nemirovski uncertainty set becomes a conic quadratic program. Bandi and Berstimas (2012) develop a new approach to constructing uncertainty sets using conclusions from probability. The approach incorporates distributional information which can be estimated with historical data and the CLT is assumed to hold which is used to construct the uncertainty sets.

The models discussed above are static models where all decisions are "here and now" decisions that are made before uncertainties are realized. Ben-Tal et al. (2004) present a dynamic approach called adjustable RO (ARO) approach where "wait and see" decisions are made after uncertainty realizations occur. This approach can produce less conservative solutions than the static approach but it may be computationally intractable. The authors formulate an affinely adjustable RO (AARO) approach (this is also called the linear decision rule approach in the literature) by restricting the decisions to be affine function of uncertain data which is shown to be tractable for certain cases.

With the exception of Soyster (1973), the papers previously discussed have considered row-wise uncertainty sets. However, column-based uncertainty sets are still important since they can represent uncertainty in processes (Soyster and Murphy 2013). Since it is straightforward that the dual formulation corresponding to a robust problem with row-wise uncertainty has column-based uncertainty, there has been a stream of research investigating this relationship. Minoux (2009) considers robust linear programming with right-hand side uncertainty, a special case of column-wise uncertainty. The main results are that the dual of the robust model 
is not equivalent to the robust version of the dual, and the formulation of a twostage approach produces less conservative solutions for column-wise robust problems than the Soyster model. Beck and Ben-Tal (2009) show that the dual of the robust counterpart is the same as the optimistic counterpart of the dual, where the optimistic counterpart is defined as a solution to a problem that satisfies the constraints for at least one realization in the uncertainty set. Minoux (2011) further investigates the two-stage approach to robust linear programming under righthand side uncertainty which is shown to be NP-hard for the general case and the author shows several polynomially solvable special cases.

A robust optimization problem can be solved using either a direct reformulation approach or what is called an adversarial approach. First we describe the various solution approaches utilizing a direct reformulation. Soyster (1973) shows that the robust counterpart of a linear program under column-wise, ellipsoidal uncertainty is a linear program. Ben-Tal and Nemirovski (1999) show that the robust counterpart of a linear program under row-wise, ellipsoidal uncertainty is a conic quadratic program. Bertsimas and Sim (2004) show that the robust counterpart under budget uncertainty (polyhedral) remains a linear program.

A cutting plane approach is proposed in Bienstock and Ozbay (2008) which involves solving the robust problem over a finite set of uncertainty scenarios. The worst-case uncertainty scenario for that problem is then identified by solving an adversarial problem and added to the set and then the problem is re-solved using the updated set of uncertainty scenarios. This process continues in an iterative fashion until the robust solution is obtained.

\subsection{RO: Supply Chain Applications Under Demand Uncertainty}

A large proportion of the literature on RO applied to supply chain problems deals with demand uncertainty. For example, Ben-Tal et al. (2005) solves the two-echelon, multi-period retailer-supplier flexible commitment contract problem under demand uncertainty using the AARO approach. Also using the AARO approach, Ang et al. (2012) examine the storage assignment problem in unit-load warehouses under demand uncertainty. Zhang (2011) shows that two-stage minimax regret robust uncapacitated lot-sizing problems are polynomially solvable when demand uncertainty is characterized using interval uncertainty sets.

Over the past decade there have been many papers that have applied RO to problems in inventory control. Bertsimas and Thiele (2006) develop an RO approach for inventory control where demand is uncertain using a budget uncertainty set first derived in Bertsimas and Sim (2004). The approach involves applying RO to a reformulation of the inventory problem. Recently, Gorissen and Den Hertog (2013) discuss the conservatism of such a formulation. Despite the conservatism many papers take this approach in applying RO to problems in operations management (e.g., Ben-Tal et al. 2005, José Alem and Morabito 2012, Wei et al. 2011, Aouam and Brahimi 2013). Bienstock and Ozbay (2008) alleviate this conservatism for an inventory problem under demand uncertainty and solve it using a Benders' decomposition approach. Rikun (2011) extends this by considering a multi-echelon system with more cost structure as well as a polyhedral uncertainty set motivated by the CLT. 
Wei et al. (2011) formulate a robust production planning problem with uncertain demands and returns using a RO model with budget uncertainty sets. José Alem and Morabito (2012) examine a production planning problem for a furniture company with uncertain costs and demands using a RO model with budget uncertainty sets. Aouam and Brahimi (2013) formulate a static RO model for a production planning problem with order acceptance decisions under demand uncertainty using the budget uncertainty set approach. Carlsson et al. (2014) study the distribution and inventory planning problem for a large pulp producer using a RO model with budget uncertainty sets.

\subsection{RO: Supply Chain Applications Under Supply Uncertainty}

In the above papers involving demand uncertainty, the supply-side is assumed to be deterministic and order lead times are assumed to be either zero or fixed. There have been far fewer papers on robust optimization with supply uncertainty, and they mainly deal with yield uncertainty and raw material supply uncertainty. Bohle et al. (2010) develop a static RO model using the budget uncertainty set approach for wine grape harvesting scheduling where productivity of the manual harvesting method (i.e., yield) is uncertain. Alvarez and Vera (2011) formulate a static RO model for a sawmill planning problem with yield uncertainty using the budget uncertainty set approach. Similarly, Varas et al. (2014) formulate a static RO model for a sawmill planning problem under demand and raw material supply uncertainty using the budget uncertainty set approach. Movahed and Zhang (2013) present a scenario-based robust approach to compute $(s, S)$ parameters for an inventory problem under demand and lead time uncertainty, and we emphasize that the modeling approach (scenario-based RO) is based on Mulvey et al. (1995), which is a different methodology than the set-based RO approach considered in this paper.

In this paper, we develop several RO models for a multi-period inventory problem under both demand and lead time uncertainty (which lies in the recourse matrix) where the uncertainty set is binary and we develop a solution method based on Benders' decomposition to solve it.

\section{Robust Optimization}

In this section we describe the RO methodology and several uncertainty sets that are used in this paper. Consider the following nominal linear program:

$$
\begin{aligned}
& \min _{\mathbf{x}} \mathbf{c}^{\top} \mathbf{x} \\
& \text { subject to }
\end{aligned}
$$

$\mathbf{A x} \leq \mathbf{b}$ 
where the decision variable is $\mathbf{x} \in \mathbb{R}^{n}$, and parameters are $\mathbf{A} \in \mathbb{R}^{m \times n}, \mathbf{c} \in \mathbb{R}^{n}$, and $\mathbf{b} \in \mathbb{R}^{m}$. The robust version of Formulation (1) is the following:

$$
\min _{\mathbf{x}} \max _{(\mathbf{A}, \mathbf{b}, \mathbf{c}) \in \mathbf{U}} \mathbf{c}^{\top} \mathbf{x}
$$

subject to

$$
\mathbf{A x} \leq \mathbf{b} \quad \forall(\mathbf{A}, \mathbf{b}, \mathbf{c}) \in \mathbf{U}
$$

where $\mathbf{U}$ is an uncertainty set. The solution to Formulation (2) remains feasible for any realization of data uncertainty within $\mathbf{U}$, and it achieves the best worstcase objective value. This is a semi-infinite optimization problem because there are infinitely many constraints, however for many uncertainty sets the problem can be reformulated to be tractably solvable (these reformulations are often called "robust counterparts" in the literature). Next, several uncertainty sets are described.

\subsection{Budget uncertainty sets}

Budget uncertainty sets were first introduced in Bertsimas and Sim (2004). Without loss of generality, consider a problem where the only uncertain parameters are the elements of $\mathbf{A}$ where $a_{i j} \in\left[\bar{a}_{i j}-\hat{a}_{i j}, \bar{a}_{i j}+\hat{a}_{i j}\right]$. Define the scaled deviation from the nominal value as $z_{i j}=\left(a_{i j}-\bar{a}_{i j}\right) / \hat{a}_{i j}$, so that $\left|z_{i j}\right| \leq 1$. The cumulative deviation from the nominal value for each row $i$ is bounded by a budget parameter, that is, $\sum_{j=1}^{n} z_{i j} \leq \Gamma_{i}$. The budget uncertainty set is the following:

$$
U_{i}^{b}=\left\{\left(a_{i 1}, \ldots, a_{i n}\right):\left|z_{i j}\right| \leq 1 \forall j, \sum_{j=1}^{n} z_{i j} \leq \Gamma_{i}\right\} \quad i=1, \ldots, m
$$

An advantage of this uncertainty set is that the robust counterpart of a linear program can be reformulated as a linear program (see Bertsimas and Sim (2004) for details). A disadvantage of this method is that it is unclear what value $\Gamma_{i}$ should take. One approach is to show the full spectrum of setting the budget from $\Gamma_{i}=0$ (which is equivalent to the nominal formulation) to $\Gamma_{i}=J_{i}$, where $J_{i}$ is the maximum number of parameters in row $i$ that may vary using sensitivity analysis (Bertsimas and Thiele 2006, José Alem and Morabito 2012). This allows the decision maker to examine the robustness tradeoff, or "the price of robustness" (Bertsimas and Sim 2004). Another approach is to set the upper and lower bounds of the $a_{i j}$ parameters according to a $(1-\alpha) \%$ confidence interval and then set the budget parameter to a low value if the confidence interval is very wide since it is unlikely that many of the $a_{i j}$ 's will reach their bounds, or set the budget parameter high if the confidence interval is narrower (Denton et al. 2010).

As a remark, this approach does not require distributional information or past data, and if these are available it is not straightforward how to use such information.

\subsection{Central limit theorem-based uncertainty sets}

The CLT-based uncertainty set is introduced in Bandi and Berstimas (2012) where the authors replace the axioms of probability theory and the concept of random 
variables with uncertainty sets derived from conclusions of probability, in particular the Central Limit Theorem. Consider i.i.d. random variables $X_{i}, i=1, \ldots, n$ with mean $\mu$ and variance $\sigma^{2}$, and define the sum $Y_{n}=\sum_{i=1}^{n} X_{i}$. The Central Limit Theorem states that, as $n \rightarrow \infty,\left(Y_{n}-n \mu\right) / \sigma \sqrt{n}$ is asymptotically distributed as a standard normal variable. The CLT-based uncertainty set is based on the z-test where the inputs to this z-test are the uncertainty realizations. The CLT-based uncertainty set is the following:

$$
U^{C L T}=\left\{\left(X_{1}, \ldots, X_{n}\right):\left|\sum_{i=1}^{n} X_{i}-n \mu\right| \leq \Gamma \sigma \sqrt{n}\right\}
$$

Using standard normal tables we can find probabilities, e.g.,

$$
\mathbb{P}\left(\left|\left(Y_{n}-n \mu\right) / \sigma \sqrt{n}\right| \leq 2\right) \approx 0.95
$$

so the $\Gamma$ parameter can be specified by the modeler to satisfy some asymptotic probability guarantee (e.g., in this example $\Gamma=2$, satisfies a $95 \%$ guarantee). This property is the main advantage of this uncertainty set. Also, like the budget uncertainty set, the CLT-based uncertainty set is polyhedral which allows the robust counterpart of a linear program to be formulated as a linear program. The disadvantage of this method is that the modeler must assume the data belongs to a specific probability distribution, and furthermore, the approach does not provide a finite-sample probability guarantee.

Also note that the budget uncertainty set is directly applied to the $\mathbf{A}$ matrix of the uncertain problem while the CLT-based uncertainty set is applied to parameters which behave like a sequence of i.i.d. random variables. While this approach can easily be applied to problems such as the inventory control problem in this paper where there is a sequence of demands and lead times, it will not be applicable in general, as is the budget approach.

\section{Robust Multi-Period Inventory Model}

The problem setup is as follows. We consider a periodic review inventory control problem for a single facility over a finite time horizon of $T$ time periods. The planner places an order, $u_{i}$, at the beginning of the time period $i$ which incurs a variable $\operatorname{cost} c$. Then demand, $d_{i}$, occurs during the period. Then order $u_{i}$ arrives if the lead time is zero; if the lead time is $L T(i)$, then $u_{i}$ arrives in $L T(i)$ periods. At the end of each period costs are incurred for holding positive inventory (holding cost, $h$ ), or for negative inventory (backorder cost, $b$ ). The initial inventory level is $x_{0}$.

The lead time parameter $\delta_{i k}$ is defined as follows:

\section{Definition 1}

$$
\delta_{i k}= \begin{cases}0 & L T(i)>k-i \\ 1 & \text { otherwise }\end{cases}
$$

where $L T(i)$ represents the lead time (measured in integer number of periods) of the order in period $i$, where $i=1, \ldots, T$ and $k \geq i$. If $\delta_{i k}=1$, the order placed in period $i$ has arrived by period $k$. If $\delta_{i k}=0$, the order placed in period $i$ has not yet arrived by period $k$. 
Assuming fixed demand and fixed lead time, the inventory level at the end of period $t$ is $x_{t+1}$, and the inventory balance constraints are the following:

$$
x_{t+1}=x_{0}+\sum_{i=1}^{t}\left(\delta_{i t} u_{i}-d_{i}\right)
$$

The inventory problem is to minimize the total cost $Z$ which can be expressed as follows:

$$
Z=\min _{u \geq 0}\left\{\sum_{t=1}^{T}\left(c u_{t}+\max \left\{h x_{t+1},-b x_{t+1}\right\}\right)\right\}
$$

Problem (7) is nonlinear because of the max function in the objective. It is equivalent to the following linear program (called the epigraph formulation):

$$
\begin{aligned}
& Z=\min _{u, y}\left\{\sum_{t=1}^{T}\left(c u_{t}+y_{t}\right)\right\} \\
& \text { subject to } \\
& y_{t} \geq h\left(x_{0}+\sum_{i=1}^{t}\left(\delta_{i t} u_{i}-d_{i}\right)\right), t=1, \ldots, T \\
& y_{t} \geq-b\left(x_{0}+\sum_{i=1}^{t}\left(\delta_{i t} u_{i}-d_{i}\right)\right), t=1, \ldots, T \\
& u_{t} \geq 0, t=1, \ldots, T
\end{aligned}
$$

The solution of this problem when demand is known and lead times are zero is trivial where $u_{t}$ is set to $d_{t}$ for each time period $t$. However, when lead times and demand are uncertain the solution is unclear. In this paper we model the uncertainty by allowing the demand and lead time of each order to belong to uncertainty sets and solving a min-max problem.

\subsection{Demand uncertainty sets}

We model demand using the budget uncertainty set as follows:

$$
\begin{aligned}
\mathcal{D}_{t}^{b}=\left\{\mathbf{d} \in \mathbb{R}^{t}, \mathbf{z} \in \mathbb{R}^{t}:\right. & d_{i}=\bar{d}_{i}+z_{i} \tilde{d}_{i}, i=1, \ldots, t ; \\
& -1 \leq z_{i} \leq 1, i=1, \ldots, t ; \\
& \left.\sum_{i=1}^{t}\left|z_{i}\right| \leq \Gamma_{t},\right\} \quad t=1, \ldots, T
\end{aligned}
$$

The budget parameter $\Gamma_{t}$ is set to $\alpha t$ where $\alpha \in[0,1]$. When $\alpha=0$ the uncertainty set is reduced to $d_{t}=\bar{d}_{t}$ for all $t$. When $\alpha=1$ the demand uncertainty set is most conservative, allowing all demands to reach their worst-case values simultaneously. 
4.2 Lead time uncertainty sets

For our first model of lead time uncertainty of the order placed at time period $t$, we use the following uncertainty set:

$$
\begin{aligned}
\mathcal{L}_{t}=\left\{\delta_{t} \in \mathbb{R}^{T-t}:\right. & 0 \leq \delta_{t i} \leq 1, i=t, \ldots, T \\
& \delta_{t i} \leq \delta_{t, i+1}, i=1, \ldots, T-t-1 ; \\
& \left.\delta_{t, t+L T_{\max }}=1\right\} \quad t=1, \ldots, T
\end{aligned}
$$

where $\delta_{t}:=\left(\delta_{t t}, \ldots, \delta_{t T}\right)$. Combining the lead time uncertainty sets for all time periods, the lead time uncertainty set can be stated as

$$
\mathcal{L}=\left\{\delta_{t} \in \mathcal{L}_{t}: \quad t=1, \ldots, T\right\}
$$

The uncertainty set allows the possible realizations of uncertain order lead times at time period $t$ to be between zero and $L T_{\max }$. A major structural difference between this uncertainty set and the demand budget uncertainty set is that $\mathcal{L}_{t}$ is a column-wise set, while the demand uncertainty set is row-wise. As another remark about the set $\mathcal{L}_{t}$, note that this uncertainty set relaxes the binary structure of the $\delta_{i j}$ terms (as originally defined in Definition 1). The implication of this relaxation is that partial orders may be delivered. For example, $\delta_{11}=0.5$ means that half of the order that was placed in period 1 is delivered with zero lead time. In many supply chains partial deliveries may occur if a supplier cannot fulfill the entire order on time and ships a portion early or if a supplier has longer lead times and the customer uses an alternative supplier to satisfy immediate needs. In order to use standard robust optimization techniques involving duality to derive a robust counterpart it is required that the uncertainty sets are convex. Therefore, we introduce the lead time parameter relaxation into the uncertainty set instead of imposing binary values.

4.3 Robust Counterpart of Inventory Control Problem Under Uncertain Demand and Lead Time

The robust problem involves solving the epigraph formulation where the constraints must hold for any realization of uncertainty:

$$
Z=\min _{\mathbf{u}, \mathbf{y}}\left\{\sum_{t=1}^{T}\left(c u_{t}+y_{t}\right)\right\}
$$

subject to

$$
\begin{aligned}
& y_{t} \geq h\left(x_{0}+\sum_{i=1}^{t}\left(\delta_{i t} u_{i}-d_{i}\right)\right), t=1, \ldots, T ; \forall d \in \mathcal{D}_{t}^{b}, \forall \delta_{i} \in \mathcal{L}_{i}, i=1, \ldots, t \\
& y_{t} \geq-b\left(x_{0}+\sum_{i=1}^{t}\left(\delta_{i t} u_{i}-d_{i}\right)\right), t=1, \ldots, T ; \forall d \in \mathcal{D}_{t}^{b}, \forall \delta_{i} \in \mathcal{L}_{i}, i=1, \ldots, t \\
& u_{t} \geq 0, t=1, \ldots, T
\end{aligned}
$$

We now show that the inventory problem under demand uncertainty and lead time uncertainty can also be formulated as a linear program. 
Proposition 1 The robust counterpart of Model (12) is the following linear program:

$$
Z=\min _{\mathbf{u}, \mathbf{y}}\left\{\sum_{t=1}^{T}\left(c u_{t}+y_{t}\right)\right\}
$$

subject to

$$
\begin{aligned}
& y_{k} \geq h\left(x_{0}-\sum_{i=1}^{k} \bar{d}_{i}+\sum_{i=1}^{k} s_{i k}^{H}-\sum_{i \leq k-L T_{\max }} v_{i k}^{H}+\Gamma_{k} q_{k}+\sum_{i=1}^{k} r_{i k}\right), k=1, \ldots, T \\
& y_{k} \geq-b\left(x_{0}-\sum_{i=1}^{k} \bar{d}_{i}-\sum_{i=1}^{k} s_{i k}^{B}+\sum_{i \leq k-L T_{\max }} v_{i k}^{B}-\Gamma_{k} q_{k}-\sum_{i=1}^{k} r_{i k}\right), k=1, \ldots, T \\
& q_{k}+r_{i k} \geq \tilde{d}_{i}, \quad k=1, \ldots, T, \quad i=1, \ldots, k \\
& s_{i k}^{H}-v_{i k}^{H} \geq u_{i}, \quad k=1, \ldots, T, \quad i \leq k-L T_{\max } \\
& s_{i k}^{H} \geq u_{i}, \quad k=1, \ldots, T, \quad i>k-L T_{\max } \\
& s_{i k}^{B}-v_{i k}^{B} \geq-u_{i}, \quad k=1, \ldots, T, \quad i \leq k-L T_{\max } \\
& s_{i k}^{B} \geq-u_{i}, \quad k=1, \ldots, T, \quad i>k-L T_{\max } \\
& q_{k}, u_{k} \geq 0, \quad k=1, \ldots, T \\
& v_{i k}^{H}, v_{i k}^{B} \geq 0, \quad k=1, \ldots, T, \quad i \leq k-L T_{\max } \\
& s_{i k}^{H}, s_{i k}^{B}, r_{i k} \geq 0, \quad k=1, \ldots, T, \quad i=1, \ldots, k
\end{aligned}
$$

Proof In order for the holding constraint in Model (12) at time period $k$ to be feasible for any demand or lead time realization within the respective uncertainty set, it must satisfy

$$
\begin{aligned}
y_{k} & \geq \max _{d \in \mathcal{D}_{k}^{b}, \delta \in \mathcal{L}} h\left(x_{0}+\sum_{i=1}^{k}\left(\delta_{i k} u_{i}-d_{i}\right)\right) \\
& =h x_{0}+h\left(\max _{\delta \in \mathcal{L}} \sum_{i=1}^{k}\left(\delta_{i k} u_{i}\right)+\max _{d \in \mathcal{D}_{k}^{b}} \sum_{i=1}^{k}\left(-d_{i}\right)\right) \\
& =h\left(x_{0}-\sum_{i=1}^{k} \bar{d}_{i}\right)+h\left(\max _{\delta \in \mathcal{L}} \sum_{i=1}^{k}\left(\delta_{i k} u_{i}\right)+\max _{d \in \mathcal{D}_{k}^{b}} \sum_{i=1}^{k}\left(-z_{i} \tilde{d}_{i}\right)\right)
\end{aligned}
$$

Bertsimas and Thiele (2006) show that the robust inventory problem under demand uncertainty (using budget uncertainty sets) can be formulated as a linear program by applying principles of duality to the demand subproblem. The demand subproblem for this holding constraint $k$ is the following linear program (dual variables in parentheses): 
$\max _{z} \sum_{i=1}^{k} z_{i} \tilde{d}_{i}$

subject to

$z_{i} \leq 1, \quad i=1, \ldots, k \quad\left(r_{i k}\right)$

$\sum_{i=1}^{k} z_{i} \leq \Gamma_{k} \quad\left(q_{k}\right)$

$z_{i} \geq 0, i=1, \ldots, k$

Its dual is the following:

$$
\min _{q, r} \Gamma_{k} q_{k}+\sum_{i=1}^{k} r_{i k}
$$

subject to

$$
\begin{aligned}
& q_{k}+r_{i k} \geq \tilde{d}_{i}, \quad i=1, \ldots, k \\
& q_{k} \geq 0, r_{i k} \geq 0, \quad i=1, \ldots, k ;
\end{aligned}
$$

Similarly, the lead time subproblem for holding constraint $k$ is the following linear program (dual variables in parentheses).

$$
\begin{array}{rlr}
\max _{\delta} \sum_{i=1}^{k} \delta_{i k} u_{i} & \\
\text { subject to } & \\
\delta_{i k} & \leq 1, \quad i=1, \ldots, k & \left(s_{i k}^{H}\right) \\
-\delta_{i k} & \leq-1, \quad i \leq k-L T_{\max } & \left(v_{i k}^{H}\right) \\
\delta_{i k} & \geq 0, \quad i=1, \ldots, k &
\end{array}
$$

Formulation (17) involves the lead time parameters $\delta_{1 k}, \ldots, \delta_{k k}$. Thus the constraints of type $\delta_{t i} \leq \delta_{t, i+1}$ from the lead time uncertainty set (10) are not involved in this subproblem. The dual of this problem is

$$
\begin{gathered}
\min _{s, v}\left(\sum_{i=1}^{k}\left(s_{i k}\right)-\sum_{i \leq k-L T_{\max }}\left(v_{i k}\right)\right) \\
\text { subject to } \\
s_{i k}^{H}-v_{i k}^{H} \geq u_{i}, \quad i \leq k-L T_{\max } \\
s_{i k}^{H} \geq u_{i}, \quad i>k-L T_{\max } \\
v_{i k}^{H} \geq 0, \quad i \leq k-L T_{\max } \\
s_{i k}^{H} \geq 0, \quad i=1, \ldots, k
\end{gathered}
$$

The lead time subproblem for backorder constraint $k$ is similar. We substitute the duals for these subproblems back into the original problem and obtain the robust counterpart, Formulation (13). 


\section{Solution Methods}

Two approaches are used to solve this problem in this paper. First, the epigraph reformulation (ER) approach involves solving Model (13) directly using an optimization solver such as CPLEX or GUROBI which can be solved quickly since it is a linear programming problem. However, it may produce solutions that are too conservative. Recall that Model (13) is equivalent to Model (12), which is just the epigraph formulation of Model (7) under uncertainty. As discussed by Gorissen and Den Hertog (2013), the conservatism occurs because of the structure of the epigraph reformulation which bring the sum terms from Model (7) to the constraints. Then, the worst-case values of each of the terms in the sum from the original objective function (Model $(7)$ ) can occur at different realizations of the uncertain parameter.

To find less conservative solutions we solve the "true min-max problem" using the Adversarial approach. The "true min-max problem" is the following:

$$
\min _{u \geq 0}\left\{\max _{\delta \in \mathcal{L}, d \in \mathcal{D}^{b}} \sum_{t=1}^{T}\left(c u_{t}+\max \left\{h \tilde{x}^{t},-b \tilde{x}^{t}\right\}\right)\right\}
$$

where $\delta:=\left\{\delta_{i j}: i=1, \ldots, T, j=i, \ldots, T\right\}$ and $\tilde{x}^{t}=x_{0}+\sum_{i=1}^{t}\left(\delta_{i t} u_{i}-d_{i}\right)$

This "true min-max problem" solves the unreformulated problem, Model (7), under uncertainty. Model (19) is clearly nonconvex, as it involves maximizing a convex function over a convex set, in contrast to Model (13), which is a linear program. Model (19) is solved under two ordering policies: static (called static because the ordering decisions for the entire time horizon are determined at time zero), and basestock. For the basestock, the ordering policy is restricted to the form

$$
u_{i}= \begin{cases}\sigma-x_{i} & x_{i}<\sigma \\ 0 & \text { otherwise }\end{cases}
$$

where $\sigma$ is the order-up-to level, and $x_{i}$ is the inventory position at time $i$.

The Adversarial approach involves maintaining a finite list of lead time and demand scenarios and minimizing the maximum cost over these scenarios in the Decision Maker Subproblem (DM). Worst-case lead time and demand scenarios are generated in the Adversarial subproblem (AP), which are used as cuts in the DM.

Algorithm 1 is a high level description of the approach. For the problem discussed in this paper, the DM is formulated as a linear program for the static policy and a mixed-integer linear program (MILP)for the basestock policy, and the AP is formulated as a MILP program for both static and basestock policies.

The number of cuts needed to converge is critical and there is no guarantee since in theory, all extreme points of the uncertainty set could be required to be generated before convergence. However, we present numerical results in Section 7 that show that instances can be solved in a reasonable time for time horizons up to $T=40$. 


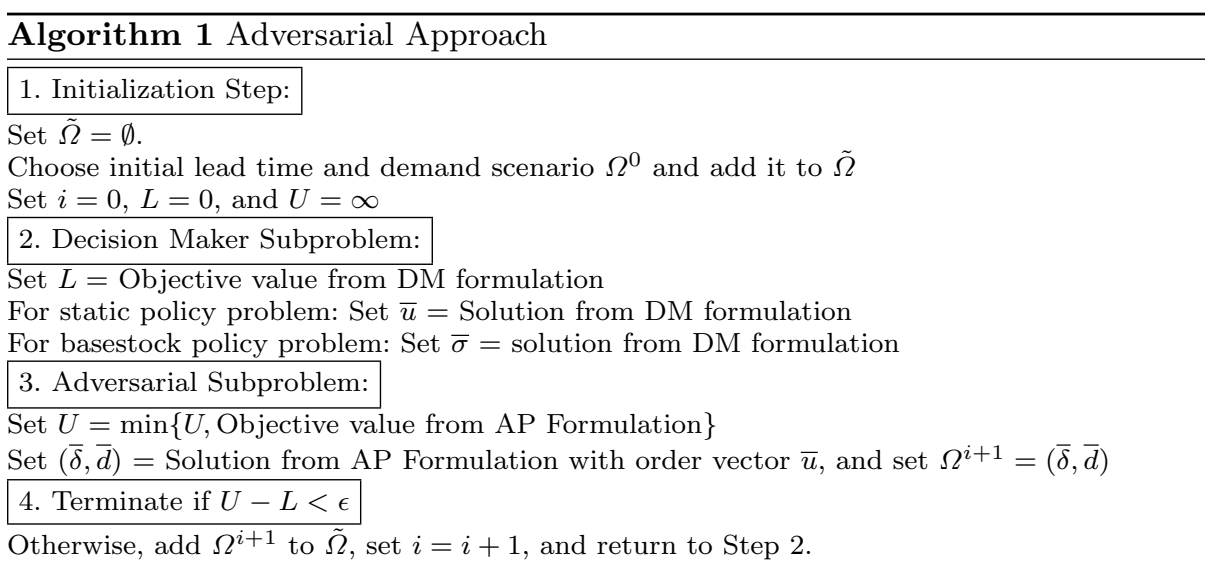

5.1 Benders' Subproblems for Static Policy

For the static policy, the explicit formulations of the Decision Maker Subproblem and Adversarial Subproblem are the following. The Decision Maker Subproblem for the static policy is the following linear program:

$\min Z$

$$
\begin{array}{ll}
\text { s.t. } & Z \geq \sum_{i=1}^{T}\left(c u_{i}+y_{i, \omega}\right) \quad \omega=0, \ldots,|\tilde{\Omega}| \\
& y_{i, \omega} \geq h\left(x_{0}+\sum_{i=1}^{t}\left(\delta_{i t}^{\omega} u_{i}-d_{i}^{\omega}\right)\right) \quad i=1, \ldots, T, \omega=0, \ldots,|\tilde{\Omega}| \\
& y_{i, \omega} \geq-b\left(x_{0}+\sum_{i=1}^{t}\left(\delta_{i t}^{\omega} u_{i}-d_{i}^{\omega}\right)\right) \quad i=1, \ldots, T, \omega=0, \ldots,|\tilde{\Omega}| \\
& u_{i} \geq 0 \quad i=1, \ldots, T .
\end{array}
$$

The Adversarial Subproblem for the static policy is the following MILP:

$$
\begin{array}{ll} 
& \max _{\delta, I, B, p, d} \sum_{i=1}^{T}\left(c \bar{u}_{i}+I_{i}+B_{i}\right) \\
\text { s.t. } \quad & I_{k} \geq h\left(x_{0}+\sum_{i=1}^{k}\left(\delta_{i k} \bar{u}_{i}-d_{i}\right)\right) \quad k=1, \ldots, T \\
& I_{k} \leq h\left(x_{0}+\sum_{i=1}^{k}\left(\delta_{i k} \bar{u}_{i}-d_{i}\right)\right)+M_{k}\left(1-p_{k}\right) \quad k=1, \ldots, T \\
& I_{k} \leq M_{k}\left(p_{k}\right) \quad k=1, \ldots, T \\
& B_{k} \geq-b\left(x_{0}+\sum_{i=1}^{k}\left(\delta_{i k} \bar{u}_{i}-d_{i}\right)\right) \quad k=1, \ldots, T
\end{array}
$$




$$
\begin{aligned}
& B_{k} \leq-b\left(x_{0}+\sum_{i=1}^{k}\left(\delta_{i k} \bar{u}_{i}-d_{i}\right)\right)+M_{k}\left(p_{k}\right) \quad k=1, \ldots, T \\
& B_{k} \leq M_{k}\left(1-p_{k}\right) \quad k=1, \ldots, T \\
& \delta_{i k} \in \mathcal{L} \quad i=1, \ldots, T, \quad k \geq i \\
& d_{i} \in \mathcal{D}_{i}^{b}, \quad i=1, \ldots, T \\
& p_{k} \in\{0,1\}
\end{aligned}
$$

where $M_{k}$ is a sufficiently large constant. $M_{k}$ should be set to a value which is always as large as $x_{k}$ can become, and as large as $-x_{k}$ can become. Therefore we can set $M_{k}=\max \{\bar{X}, \underline{X}\}$, where

$$
\begin{aligned}
& \bar{X}=x_{0}+\sum_{i=1}^{k}\left(\bar{u}_{i}-\left(\bar{d}_{i}-\tilde{d}_{i}\right)\right) \\
& \underline{X}=-\left(x_{0}+\sum_{i=L T_{\max }+1}^{k}\left(\bar{u}_{i-L T_{\max }}\right)-\sum_{i=1}^{k}\left(\bar{d}_{i}+\tilde{d}_{i}\right)\right)
\end{aligned}
$$

$\bar{X}$ represents the case where all demand realizations reach their lower bounds and all orders are received on time, maximizing the on-hand inventory, and $\underline{X}$ represents the case where all demands reach their upper bounds and orders are outstanding.

\subsection{Benders' Subproblems for Basestock Policy}

For the basestock policy, the explicit formulations of the Decision Maker Subproblem and Adversarial Subproblem are the following. The Decision Maker Subproblem for the basestock policy is the following MILP:

$\min Z$

$$
\begin{array}{ll}
\text { s.t. } & Z \geq \sum_{i=1}^{T}\left(c u_{i}^{\omega}+y_{i, \omega}\right) \quad \omega=0, \ldots,|\tilde{\Omega}| \\
& y_{i, \omega} \geq h\left(x_{0}+\sum_{i=1}^{t}\left(\delta_{i t}^{\omega} u_{i}^{\omega}-d_{i}^{\omega}\right)\right) \quad i=1, \ldots, T, \omega=0, \ldots,|\tilde{\Omega}| \\
& y_{i, \omega} \geq-b\left(x_{0}+\sum_{i=1}^{t}\left(\delta_{i t}^{\omega} u_{i}^{\omega}-d_{i}^{\omega}\right)\right) \quad i=1, \ldots, T, \omega=0, \ldots,|\tilde{\Omega}| \\
& u_{i}^{\omega} \geq 0 \quad i=1, \ldots, T, \omega=0, \ldots,|\tilde{\Omega}| \\
& u_{i}^{\omega} \leq M z_{i, \omega} \quad i=1, \ldots, T, \omega=0, \ldots,|\tilde{\Omega}| \\
& \sigma-\bar{x}_{i}^{\omega} \leq u_{i}^{\omega} \leq \sigma-\bar{x}_{i}^{\omega}+M\left(1-z_{i, \omega}\right) \quad i=1, \ldots, T, \omega=0, \ldots,|\tilde{\Omega}| \\
& x_{k+1}^{\omega}=x_{0}+\sum_{i=1}^{k} u_{i}^{\omega}-\sum_{i=1}^{k} d_{i}^{\omega} \quad k=1, \ldots, T, \omega=0, \ldots,|\tilde{\Omega}| \\
& z_{i, \omega} \in\{0,1\} \quad i=1, \ldots, T, \omega=0, \ldots,|\tilde{\Omega}|
\end{array}
$$


In Formulation (24), where both lead time and demand are uncertain, the orders $u_{i}$ at each period $i$ are indexed by the uncertainty realization $\omega$. This is because the basestock policy, defined by Equation (20), is a dynamic policy based on inventory position which is a function of demand.

The Adversarial Subproblem for the basestock policy is the following MILP:

$$
\begin{array}{ll} 
& \max _{\delta, I, B, p, d} \sum_{i=1}^{T}\left(c u_{i}+I_{i}+B_{i}\right) \\
\text { s.t. } & u_{t} \leq \bar{\sigma}-\sum_{i<t}\left(u_{i}-d_{i}\right)+M_{t}^{1}\left(1-\xi_{t}\right), \quad t=1, \ldots, T \\
& u_{t} \geq \bar{\sigma}-\sum_{i<t}\left(u_{i}-d_{i}\right), \quad t=1, \ldots, T \\
& u_{t} \leq M_{t}^{1}\left(\xi_{t}\right), \quad t=1, \ldots, T \\
& x_{k+1}=x_{0}+\sum_{i=1}\left(\tilde{u}_{i k}-d_{i}\right), \quad k=1, \ldots, T \\
& \tilde{u}_{t k} \leq u_{t}, \quad t=1, \ldots, T, \quad k=t, \ldots, t+L T_{\max }-1 \\
& \tilde{u}_{t k} \geq u_{t}-M_{t}^{1}\left(1-\delta_{t k}\right), \quad t=1, \ldots, T, \quad k=t, \ldots, t+L T_{\max }-1 \\
& \tilde{u}_{t k}=u_{t}, \quad t=1, \ldots, T, \quad k=t+L T_{\max }, \ldots, T \\
& I_{k} \geq h\left(x_{k+1}\right) \quad k=1, \ldots, T \\
& I_{k} \leq h\left(x_{k+1}\right)+M_{k}^{2}\left(1-p_{k}\right) \quad k=1, \ldots, T \\
& I_{k} \leq M_{k}^{2}\left(p_{k}\right) \quad k=1, \ldots, T \\
& B_{k} \geq-b\left(x_{k+1}\right) \quad k=1, \ldots, T \\
& B_{k} \leq-b\left(x_{k+1}\right)+M_{k}^{2}\left(p_{k}\right) \quad k=1, \ldots, T \\
& B_{k} \leq M_{k}^{2}\left(1-p_{k}\right) \quad k=1, \ldots, T \\
& \delta_{i k} \in \mathcal{L} \quad i=1, \ldots, T \quad k \geq i \\
& d_{i} \in \mathcal{D}_{i}^{b}, \quad i=1, \ldots, T \\
& u_{k}, \tilde{u}_{i k}, I_{k}, B_{k}, x_{k} \geq 0, \quad i=1, \ldots, T, \quad k=1, \ldots, T \\
& p_{k} \in\{0,1\}, \quad k=1, \ldots, T \\
& \xi_{k} \in\{0,1\}, \quad k=1, \ldots, T \\
& \quad k \\
& \quad k
\end{array}
$$

where $M_{t}^{1}$, and $M_{t}^{2}$ are sufficiently large constants. The first, second, and third constraints are Big M constraints to force the orders to follow a basestock policy. We set $M_{t}^{1}=\bar{\sigma}+\sum_{i<t}\left(\bar{d}_{i}+\tilde{d}_{i}\right)$ for $t=1, \ldots, T$, since $u_{t}$ cannot exceed this value.

Recall that the inventory level follows Equation (6), which involves $\delta_{t k} u_{t}$ terms. As long as $\delta_{t k}$ is binary, the inventory level $x_{t}$ at each period $t$ can be expressed by the fourth through seventh constraints which determine the inventory level, $x_{t}$ at each period $t$, where the lead time parameter $\delta_{t k}$ acts as an indicator variable. Notice in the sixth constraint that when $\delta_{t k}$ is zero, then $\tilde{u}_{t k}$ is forced to zero as long as $M_{t}^{1} \geq u_{t}$. Thus, the value of $M_{t}^{1}$ mentioned above is a good choice for this constraint as well. 
The next two sets of constraints determine the inventory holding cost or backorder cost that is incurred at each time period $t$. The value of the constant $M_{t}^{2}$ is set in a similar fashion to the constant from Model (22). The next constraints enforce that the lead time parameters must belong to the lead time uncertainty set, the demand parameters must belong to the lead time uncertainty set, and the variables $p_{k}$ and $\xi_{k}$ are binary.

\subsection{On Overconservatism of the Epigraph Reformulation Approach}

We use a small problem instance to illustrate the differences between the solutions obtained with the ER approach and the Adversarial approach. Our results show that the ER approach can produce overly conservative results compared to the Adversarial approach in several ways. The results show that ER conservativeness increases for instances with nonstationary demand, increases as maximum lead time increases, and increases as the critical ratio $(b /(h+b))$ increases.

To illustrate the differences between the solutions obtained with the ER approach and the Adversarial approach, we use the following small problem instance (using a time horizon $T=10$ ). The problem instance is described as follows: Demand uncertainty is characterized using the budget demand uncertainty set $\mathcal{D}^{b}$ where the average demand $\bar{d}_{t}=15$ and $\tilde{d}_{t}=5$ at each time period $t$. The costs are $h=5, b=10$, and $c=1$.

First consider the case where lead time is fixed at zero and the demand uncertainty budget is at the most conservative level, $\Gamma_{t}=t$. The second column of Table 1 shows the ER solution (calculated by solving Formulation (13)), and the corresponding costs for each period are shown in the third column. The Adversarial solution is shown in the fourth column, which is calculated by applying Algorithm 1 for the static policy where the Decision Maker Subproblem is Formulation (21) and Adversarial Subproblem is Formulation (22). Algorithm 1 solves this problem instance in two iterations. The two demand scenarios that were generated by Algorithm 1 are shown in the next two columns. The costs of the Adversarial solution for the two demand scenarios are shown in the next two columns. In the final column we show the cost for using the optimal Adversarial solution in the ER model.

The total cost for the ER solution (2000) is only $2.2 \%$ higher than the total cost for the Adversarial solution (1957.5), which is not surprising. Bertsimas and Thiele (2006) show that the ER approach works well for an inventory problem under demand uncertainty, when costs are stationary as they are in this example. However, the solutions themselves are quite different. In the ER approach, a constant amount is ordered per period, while in the Adversarial approach a higher amount is ordered in earlier periods tapering off in the last few periods.

Table 1 also illustrates how the ER approach can produce overly conservative solutions. By decoupling the terms of the objective function for each time period into separate constraints, the ER approach allows different realizations of uncertainty to produce the worst-case for each term. This is revealed in the last column in Table 1 which shows costs from the ER model using the optimal Adversarial solution as input. Notice that the cost of each time period is equal to the maximum costs of the two uncertainty realizations (D1 and D2), producing a total cost (3120) significantly higher than the total cost computed by the Adversarial 
Table 1 Comparing the ER (Formulation (13)) and Adversarial solution approaches for an instance with demand uncertainty and fixed lead time of zero. The Adversarial approach is comprised of the Decision Maker Subproblem (Formulation (21)) and the Adversarial Subproblem (Formulation (22))

\begin{tabular}{|c|c|c|c|c|c|c|c|c|}
\hline $\mathrm{t}$ & $\begin{array}{l}\text { ER } \\
\text { soln }\end{array}$ & $\begin{array}{l}\mathrm{ER} \\
\text { cost }\end{array}$ & $\begin{array}{l}\text { Adv } \\
\text { soln }\end{array}$ & $\begin{array}{l}\text { Scen } \\
\text { D1 }\end{array}$ & $\begin{array}{l}\text { Scen } \\
\text { D2 }\end{array}$ & $\begin{array}{l}\text { Adv } \\
\text { Cost D1 }\end{array}$ & $\begin{array}{l}\text { Adv } \\
\text { Cost D2 }\end{array}$ & $\begin{array}{l}\text { ER cost using } \\
\text { Adv soln }\end{array}$ \\
\hline 1 & 16.67 & 33.33 & 20 & 20 & 10 & 0 & 50 & 50 \\
\hline 2 & 16.67 & 66.67 & 20 & 20 & 10 & 0 & 100 & 100 \\
\hline 3 & 16.67 & 100 & 20 & 20 & 10 & 0 & 150 & 150 \\
\hline 4 & 16.67 & 133.33 & 20 & 20 & 10 & 0 & 200 & 200 \\
\hline 5 & 16.67 & 166.67 & 20 & 20 & 10 & 0 & 250 & 250 \\
\hline 6 & 16.67 & 200 & 20 & 20 & 10 & 0 & 300 & 300 \\
\hline 7 & 16.67 & 233.33 & 4.1667 & 20 & 10 & 158.33 & 270.83 & 270.83 \\
\hline 8 & 16.67 & 266.67 & 0 & 20 & 10 & 358.33 & 220.83 & 358.33 \\
\hline 9 & 16.67 & 300 & 0 & 20 & 10 & 558.33 & 170.83 & 558.33 \\
\hline 10 & 16.67 & 333.33 & 0 & 20 & 10 & 758.33 & 120.83 & 758.33 \\
\hline Total & & 2000 & & & & 1957.5 & 1957.5 & 3120.00 \\
\hline
\end{tabular}

approach (1957.5). It is clear that these two uncertainty realizations cannot occur simultaneously.

Figure 1 shows the percentage increase in the worst-case cost of the ER approach over the Adversarial approach for the same example for the full range of $\alpha$ when lead time uncertainty is also present. We characterize lead time uncertainty using the lead time uncertainty set $\mathcal{L}_{t}$ for each period $t$ and vary the parameter $L T_{\max }$ between zero and three. When costs and demands are stationary the ER solution may overestimate the worst-case solution by close to $25 \%$ when lead times are between zero and three periods. These numerical results demonstrate that the direct reformulation approach is overly conservative even when costs are stationary. The overconservatism of the ER is increased when average demand is time varying, as shown in Figure 2 which is for an example with average demand $\bar{d}_{t}$ generated from a discrete uniform distribution, $U(5,25)$.

Next, we examine an instance with parameters $T=20, c=1, h=10, \bar{d}=20$, and $\tilde{d}=10$, and we vary lead times $\left(L T_{\max }\right)$, uncertainty budget $(\alpha)$, and critical ratio $(b / b+h)$. Table 2 shows that the overconservatism may also be related to the critical ratio $(b /(h+b))$ (Zipkin 2000). The last column reveals that ER conservativeness increases on average as the critical ratio increases.

\section{Extension to More General Uncertainty Sets}

\subsection{Lead Time Uncertainty Sets: A Budget Approach}

The uncertainty sets described so far for the multi-period inventory problem include a budget-type demand uncertainty set and a lead time uncertainty set. The benefit of budget uncertainty sets is the ability to control the conservatism by varying the degree of uncertainty from the nominal case (without uncertainty) to the worst-case (high uncertainty). The lead time uncertainty set $\mathcal{L}_{t}$ for the order lead time at period $t$ does not involve budget-like constraints.

It is not straightforward how to develop a budget-type lead time uncertainty set when using the ER approach. Budget constraints require a sum of deviations 


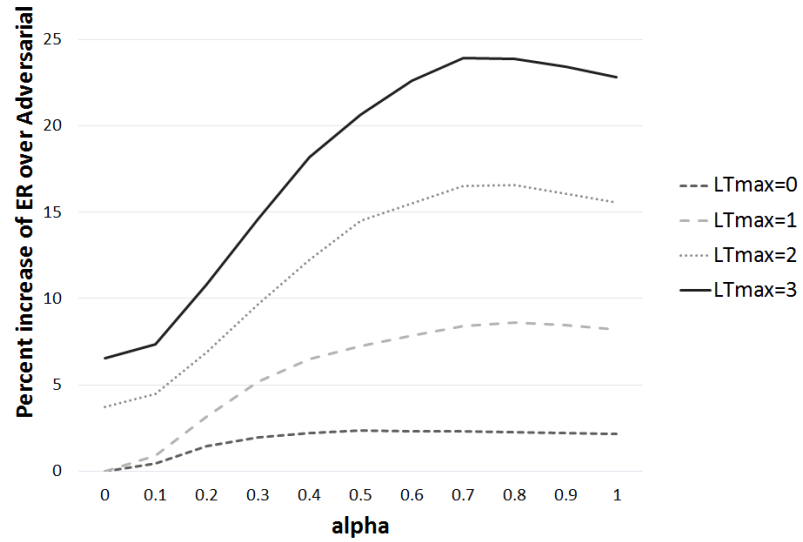

Fig. 1 The percentage of increase of ER solution over Adversarial solution for an instance with stationary demand

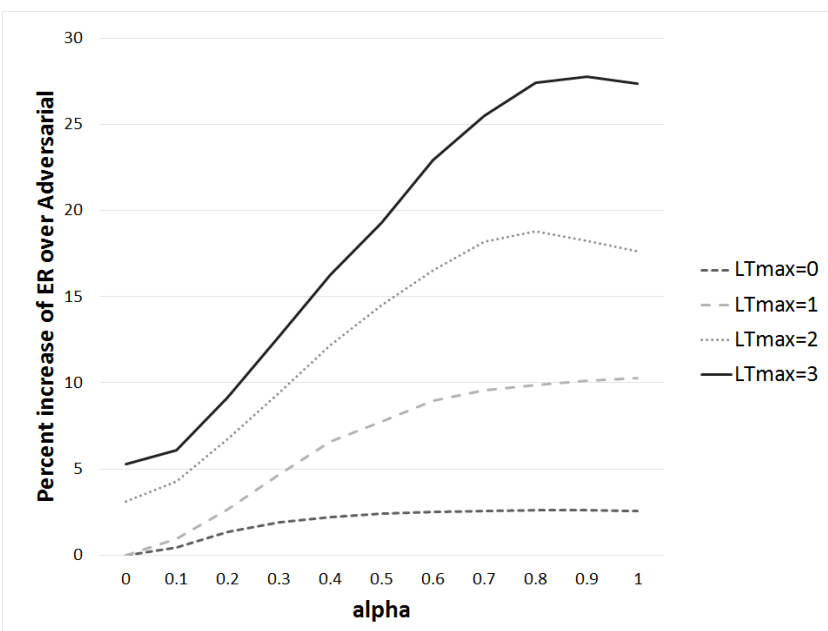

Fig. 2 The percentage of increase of ER solution over Adversarial solution for an instance with time varying demands

to be less than or equal to some budget parameter. In lead time uncertainty set $\mathcal{L}_{t}$, lead times can deviate from zero to $L T_{\max }$. Let us denote $X_{t}$ as the lead time of an order placed in period $t$. We remark that $X_{t}$ is equal to $\sum_{j=0}^{L T_{\max }}\left(1-\delta_{t, t+j}\right)$. However, Formulation (17), the subproblem for time period $k$, involves only lead time parameters $\delta_{i k}, \ldots, \delta_{k k}$ which represents whether orders at periods $1, \ldots, k$ have arrived at period $k$. Within the holding or backorder constraint for time $t$, there is not information available pertaining to the actual arrival period of previous orders. Therefore a 'budget' on a sum of deviations for $\left(X_{1}, \ldots, X_{k}\right)$ is not directly implementable for the ER approach. 
Table 2 Percent increase of ER solution over Adversarial solution for instances with parameters $T=20, c=1, h=10, \bar{d}=20$, and $\tilde{d}=10$, varying lead times $\left(L T_{\max }\right)$, uncertainty budget $(\alpha)$, and critical ratio $(b / b+h)$. The final column computes the average percent increase over all instances within the row.

\begin{tabular}{|c|c|c|c|c|c|c|c|c|c|c|c|c|c|}
\hline & & & & & & & $\alpha$ & & & & & & \\
\hline$L T_{\max }$ & $\frac{b}{(b+h)}$ & 0 & 0.1 & 0.2 & 0.3 & 0.4 & 0.5 & 0.6 & 0.7 & 0.8 & 0.9 & 1.0 & Avg \\
\hline \multirow{4}{*}{1} & $1 / 2$ & 0.0 & 0.5 & 0.6 & 0.6 & 0.6 & 0.6 & 0.6 & 0.6 & 0.5 & 0.5 & 0.5 & 0.5 \\
\hline & $2 / 3$ & 0.0 & 2.2 & 3.4 & 4.0 & 4.2 & 4.1 & 4.3 & 4.2 & 4.1 & 4.0 & 3.8 & 3.5 \\
\hline & $5 / 6$ & 1.9 & 4.6 & 6.2 & 7.3 & 8.0 & 8.8 & 9.1 & 8.3 & 7.9 & 7.7 & 7.2 & 7.0 \\
\hline & $10 / 11$ & 2.2 & 5.5 & 8.2 & 10.2 & 11.4 & 12.6 & 12.5 & 12.0 & 11.5 & 11.1 & 10.6 & 9.8 \\
\hline \multirow{4}{*}{2} & $1 / 2$ & 0.0 & 0.2 & 0.4 & 0.4 & 0.4 & 0.4 & 0.4 & 0.4 & 0.4 & 0.4 & 0.3 & 0.3 \\
\hline & $2 / 3$ & 0.0 & 1.4 & 2.2 & 2.6 & 3.0 & 3.2 & 3.5 & 3.8 & 4.2 & 4.7 & 4.9 & 3.0 \\
\hline & $5 / 6$ & 0.6 & 2.3 & 3.9 & 5.0 & 5.8 & 6.3 & 7.1 & 8.1 & 8.8 & 9.9 & 10.8 & 6.2 \\
\hline & $10 / 11$ & 1.1 & 7.7 & 4.9 & 6.3 & 7.7 & 9.1 & 10.1 & 11.4 & 13.2 & 15.5 & 17 & 9.5 \\
\hline \multirow{4}{*}{3} & $1 / 2$ & 0.0 & 0.1 & 0.2 & 0.3 & 0.3 & 0.3 & 0.3 & 0.3 & 0.3 & 0.3 & 0.3 & 0.2 \\
\hline & $2 / 3$ & 0.0 & 0.7 & 1.5 & 1.9 & 2.0 & 2.3 & 2.6 & 3.0 & 3.7 & 5.1 & 6.6 & 2.7 \\
\hline & $5 / 6$ & 0.2 & 1.0 & 2.1 & 3.0 & 3.8 & 4.3 & 5.1 & 6.3 & 8.1 & 10.1 & 14.3 & 5.3 \\
\hline & $10 / 11$ & 0.4 & 2.8 & 3.8 & 3.7 & 4.7 & 5.9 & 7.5 & 9.4 & 11.6 & 15.3 & 18.9 & 7.6 \\
\hline
\end{tabular}

However, next we present a budget-type lead time uncertainty set which can be used in the Adversarial approach since the Adversarial approach considers all uncertainties simultaneously.

Definition 2 The following is a budget-type lead time uncertainty set:

$$
\begin{aligned}
\mathcal{L}^{b}=\{ & \delta_{t} \in \mathcal{L}_{t} \quad t=1, \ldots, T: \\
& \sum_{t=1}^{\tau} \sum_{j=t}^{t+\left\lfloor\frac{L T_{\max }}{2}\right\rfloor-1} \delta_{t j} \leq \underline{\Gamma}_{\tau}, \quad \tau=1, \ldots, T \\
& \left.\sum_{t=1}^{\tau} \sum_{j=t+\left\lfloor\frac{L T_{\max }}{2}\right\rfloor}^{t+L T_{\max }-1}\left(1-\delta_{t j}\right) \leq \bar{\Gamma}_{\tau}, \quad \tau=1, \ldots, T\right\}
\end{aligned}
$$

where $\delta_{t}:=\left(\delta_{t t}, \ldots, \delta_{t T}\right)$ and $\bar{\Gamma}_{\tau}, \underline{\Gamma}_{\tau}$ are budget parameters.

For simplicity, we assume that the nominal lead time value is the integer value $\left\lfloor\frac{L T_{\max }}{2}\right\rfloor$. For the nominal case (without uncertainty), per Definition 1, all values of $\delta_{t i}$ are set to zero for $i<\left\lfloor\frac{L T_{\max }}{2}\right\rfloor+t$, and set to one for $i \geq\left\lfloor\frac{L T_{\max }}{2}\right\rfloor+t$.

The roles of the budget parameters $\bar{\Gamma}_{\tau}$ and $\underline{\Gamma}_{\tau}$ are to adjust the conservativeness of the model, as it may be unlikely that all uncertainties will reach their bounds simultaneously. The number of lead time uncertainty realizations smaller than the nominal lead time are controlled using $\underline{\Gamma}_{\tau}$, and the number of lead time uncertainty realizations larger than the nominal lead time are controlled using $\bar{\Gamma}_{\tau}$. In this study, we set $\underline{\Gamma}_{\tau}=\beta \tau\left\lfloor\frac{L T_{\max }}{2}\right\rfloor$ and $\bar{\Gamma}_{\tau}=\beta \tau\left(L T_{\max }-\left\lfloor\frac{L T_{\max }}{2}\right\rfloor\right)$ where $0 \leq \beta \leq 1$. The implication is that when $\beta=0$, all lead time parameters are forced to their nominal values. When $\beta=1$, then the uncertainty set allows all order lead times to reach their maximum values. The major value of budget uncertainty sets is to allow the decision maker to make tradeoffs between worst-case cost and robustness. 


\subsection{Uncertainty Sets: A Central Limit Theorem Based Approach}

So far, we have not used distributional information in constructing the lead time uncertainty sets. In practice, there is often historical data that can be used to estimate the distribution of orders. While stochastic optimization methods aim to use the assumed probability distribution directly, we follow the ideas set forth in Bandi and Berstimas (2012) to use conclusions of probability theory, specifically the CLT, to model the lead time uncertainty set.

The CLT states that if $X_{i}, i=1, \ldots, n$ are i.i.d. random variables with mean $\mu$ and variance $\sigma^{2}$, for a sufficiently large $n$, the random variable $Z=\sum_{i=1}^{n} X_{i}$ is distributed as a standard normal. We can use standard normal tables to compute $\mathbb{P}(|Z| \leq \Gamma)$ for a given $\Gamma$ parameter (For example, $\mathbb{P}(|Z| \leq 2)=0.95$ ). Then the CLT-uncertainty set is defined as

$$
U_{\epsilon}=\left\{\left(X_{1}, \ldots, X_{n}\right) \mid-\Gamma \sigma \sqrt{n} \leq \sum_{i=1}^{n} X_{i}-n \mu \leq \Gamma \sigma \sqrt{n}\right\}
$$

First, we examine the case where the assumed distribution of lead times is discrete uniform on the interval $[\underline{\theta}, \bar{\theta}]$. The mean and variance of the discrete uniform distribution are $\mu=\frac{\underline{\theta}+\bar{\theta}}{2}$ and $\sigma^{2}=\frac{(\bar{\theta}-\underline{\theta}+1)^{2}-1}{12}$ respectively.

Definition 3 The following is a CLT-based uncertainty set for random variables $Z_{i} \sim U\left[0, L T_{\max }\right]$, where $1 \leq i \leq T$ :

$$
U_{\epsilon}^{L T}=\left\{\left(Z_{1}, \ldots, Z_{T}\right) \mid T \hat{\mu}-\hat{\sigma} \sqrt{T} \Gamma_{\epsilon}^{L T} \leq \sum_{i=1}^{T} Z_{i} \leq T \hat{\mu}+\hat{\sigma} \sqrt{T} \Gamma_{\epsilon}^{L T}\right\}
$$

where $Z_{i}$ is the lead time of order $i, \hat{\mu}=L T_{\max } / 2, \hat{\sigma}=\sqrt{\frac{L T_{\max }^{2}+2 L T_{\max }}{12}}$, and $\Gamma_{\epsilon}^{L T}$ is chosen empirically.

Using the lead time parameter of our model, we define

$$
Z_{i}=\sum_{j=i}^{\min \left\{T, i+L T_{\max }-1\right\}}\left(1-\delta_{i j}\right)
$$

where the min function in the upper bound of the summation is due to imposing the requirement that all orders are received by the end of the time horizon. The relationship between $Z_{i}$ and $\delta_{i j}$ is illustrated in Table 3 for lead time parameters $\delta_{i j}$. There is a one-to-one correspondance of $Z_{i}$ to the values of $\delta_{i j}$ parameters under binary $\delta_{i j}$ 's.

Using the relationship in Equation (29), we rewrite Equation (28) as

$$
\begin{array}{r}
U_{\epsilon}^{L T}=\left\{\delta_{i j} i=1, \ldots, T j=i, \ldots, T \mid \sum_{j=i}^{\min \left\{T, i+L T_{\max }-1\right\}}\left(1-\delta_{i j}\right) \geq T \hat{\mu}-\hat{\sigma} \sqrt{T} \Gamma_{\epsilon}^{L T}\right. \\
\left.\sum_{j=i}^{\min \left\{T, i+L T_{\max }-1\right\}}\left(1-\delta_{i j}\right) \leq T \hat{\mu}+\hat{\sigma} \sqrt{T} \Gamma_{\epsilon}^{L T}\right\}
\end{array}
$$


Table $3 Z_{i}$, defined in Equation (29) for various realizations of $\delta_{i j}$

\begin{tabular}{|c|c|c|c|c|c|c|c|}
\hline$\delta_{i, i}$ & $\delta_{i, i+1}$ & $\delta_{i, i+2}$ & $\ldots$ & $\delta_{i, i+L T_{\max }-2}$ & $\delta_{i, i+L T_{\max }-1}$ & $\delta_{i, i+L T_{\max }}$ & $Z_{i}$ \\
\hline 1 & 1 & 1 & $\ldots$ & 1 & 1 & 1 & 0 \\
0 & 1 & 1 & $\ldots$ & 1 & 1 & 1 & 1 \\
0 & 0 & 1 & $\ldots$ & 1 & 1 & 1 & 2 \\
0 & 0 & 0 & $\ldots$ & 0 & 1 & 1 & $L T_{\max }-1$ \\
0 & 0 & 0 & $\ldots$ & 0 & 0 & 1 & $L T_{\max }$ \\
\hline
\end{tabular}

Thus, we define the CLT-based lead time uncertainty set as $\mathcal{L}_{C L T}=\mathcal{L} \cap U_{\epsilon}^{L T}$. Similarly, the following is a demand uncertainty set based on the CLT.

$$
U_{\epsilon}^{d}=\left\{\left(d_{1}, \ldots, d_{T}\right) \mid T \hat{\mu}_{d}-\hat{\sigma}_{d} \sqrt{T} \Gamma_{\epsilon}^{d} \leq \sum_{i=1}^{T} d_{i} \leq T \hat{\mu}_{d}+\hat{\sigma}_{d} \sqrt{T} \Gamma_{\epsilon}^{d}\right\}
$$

where $d_{i}$ is the demand at time $t, \hat{\mu}_{d}$ and $\hat{\sigma}_{d}$ are the mean and standard deviation of demand, respectively, and $\Gamma_{\epsilon}^{d}$ is chosen empirically.

\section{Computational Results}

First we study the computational performance of the Benders' decomposition algorithm for calculating static and basestock inventory policies. All computations were carried out on a $3.5 \mathrm{GHz}$ desktop with $32 \mathrm{~GB}$ memory, and the subproblems were solved using CPLEX 12.6. From Algorithm 1, the termination criteria used was $\epsilon=0.01$, representing a $1 \%$ optimality gap. For the first experiment we considered time horizons of 10, 20, and 40 periods, and maximum lead times of 2, 4, 6 , and 8 periods, with minimum lead time equal to zero in all scenarios. We ran 50 instances for each combination of time horizon/lead time scenario, and the computation times and iteration counts are shown in Tables 4 and 5. Other parameters were set as follows: Average demand $\left(\overline{d_{t}}\right)$ was sampled from a discrete uniform distribution from 20 to $40 ; \tilde{d}_{t}$ was sampled from a discrete uniform distribution from 5 to 20; ordering cost $(c)$ was sampled from a uniform distribution from 0 to 10; holding cost $(h)$ was sampled from a discrete uniform distribution from 10 to 10 ; and backorder cost (b) was sampled from a discrete uniform distribution from 10 to 100. The budgeted lead time and demand models were solved, where $\alpha=\beta=1$. Basestock models are solved with far fewer iterations, however the DM subproblem for basestock models can be computationally burdensome for longer time horizons $(T=40)$ resulting in higher solve time per iteration, since DM is a MILP for the basestock model, and an LP for the static model.

\subsection{Results for Budget Uncertainty}

We examine the effect of changing the budget for demand $(\alpha)$ and the budget for lead times $(\beta)$ for the static model in an example where $T=10$, demand uncertainty is characterized using the budget demand uncertainty set $\mathcal{D}_{b}$ where the average demand $\bar{d}_{t}=15$ and $\tilde{d}_{t}=5$ at each time period $t$. The costs are $h=5$, $b=10$, and $c=1$. The surface plots in Figures 3, 4, and 5 show for a given $\alpha$ (resp., 
Table 4 Computational results for solving the static problem using budget uncertainty sets where $(\alpha=\beta=1)$ using Benders' decomposition including computation time (in seconds) and number of iterations

\begin{tabular}{|c|c|c|c|c|c|c|c|}
\hline$T$ & $L T_{\max }$ & Avg Time & Min Time & Max Time & Avg Iter & Min Iter & Max Iter \\
\hline \multirow{4}{*}{10} & 2 & 3.8 & 1.7 & 8.9 & 13.7 & 6 & 30 \\
\hline & 4 & 2.9 & 0.9 & 9.1 & 10.3 & 3 & 30 \\
\hline & 6 & 1.7 & 0.7 & 3.4 & 6.3 & 3 & 12 \\
\hline & 8 & 2.0 & 0.5 & 4.0 & 7.0 & 2 & 14 \\
\hline \multirow{4}{*}{20} & 2 & 21.5 & 6.5 & 137.4 & 40.7 & 14 & 219 \\
\hline & 4 & 24.7 & 11.2 & 167.7 & 44.8 & 22 & 240 \\
\hline & 6 & 21.3 & 6.9 & 123.2 & 39.4 & 14 & 184 \\
\hline & 8 & 15.2 & 2.3 & 61.1 & 28.4 & 5 & 95 \\
\hline \multirow{4}{*}{40} & 2 & 96.8 & 13.0 & 484.3 & 78.9 & 14 & 302 \\
\hline & 4 & 342.9 & 35.2 & 1760.9 & 193.8 & 36 & 687 \\
\hline & 6 & 408.7 & 62.3 & 3694.2 & 204 & 61 & 971 \\
\hline & 8 & 417.9 & 63.2 & 5022.8 & 182.5 & 60 & 998 \\
\hline
\end{tabular}

Table 5 Computational results for solving the basestock problem using budget uncertainty sets where $(\alpha=\beta=1)$ using Benders' decomposition including computation time (in seconds) and number of iterations

\begin{tabular}{|c|c|c|c|c|c|c|c|}
\hline$T$ & $L T_{\max }$ & Avg Time & Min Time & Max Time & Avg Iter & Min Iter & Max Iter \\
\hline \multirow{4}{*}{10} & 2 & 1.7 & 1.0 & 2.3 & 4.2 & 3 & 5 \\
\hline & 4 & 1.6 & 1.0 & 2.3 & 3.8 & 3 & 5 \\
\hline & 6 & 1.3 & 0.9 & 2.7 & 3.2 & 3 & 5 \\
\hline & 8 & 1.1 & 0.6 & 1.9 & 3.0 & 2 & 4 \\
\hline \multirow{4}{*}{20} & 2 & 3.5 & 2.2 & 5.5 & 4.2 & 3 & 5 \\
\hline & 4 & 3.6 & 2.1 & 5.7 & 3.8 & 3 & 5 \\
\hline & 6 & 3.6 & 2.0 & 7.8 & 3.6 & 3 & 5 \\
\hline & 8 & 3.5 & 1.8 & 6.9 & 3.4 & 3 & 4 \\
\hline \multirow{4}{*}{40} & 2 & 10.3 & 4.9 & 21.1 & 4.4 & 3 & 7 \\
\hline & 4 & 13.9 & 4.3 & 26.5 & 4.0 & 3 & 6 \\
\hline & 6 & 39.7 & 5.6 & 1019.9 & 3.8 & 3 & 5 \\
\hline & 8 & 72.4 & 5.0 & 1031.9 & 3.7 & 3 & 5 \\
\hline
\end{tabular}

$\beta$ ), how the worst-case cost changes according to $\beta$ (resp., $\alpha$ ) for instances where the maximum order lead time $\left(L T_{\max }\right)$ equals one, two, or three periods. Table 6 shows several numerical results corresponding to Figures 3,4 , and 5 . For the case of $L T_{\max }=1$, fixing $\beta$ to 0.5 while increasing $\alpha$ from 0 to 1 increases worst-case cost by $287.3 \%$, while the worst-case cost only increases by $43.8 \%$ when $\alpha$ is fixed to 0.5 while $\beta$ was increased from 0 to 1 . However for $L T_{\max }=3$, the percentage increase in these two situations are similar (97.0\% and $107.7 \%$, respectively). This can be visualized by noticing the increasing slope of the $\beta$-axis from Figure 3 to 5 .

It is important to note that the worst-case costs determined by the optimization model shown in Figures 3, 4, and 5 could be significantly higher if the uncertainty budgets underestimate the realized uncertainty levels.

\subsection{Robust Optimization vs. Sampling Based Stochastic Programming}

Sample Average Approximation (SAA) is a stochastic programming method to minimize the expected value of a stochastic program by generating random samples and solving a deterministic program to optimize the sample average objective 


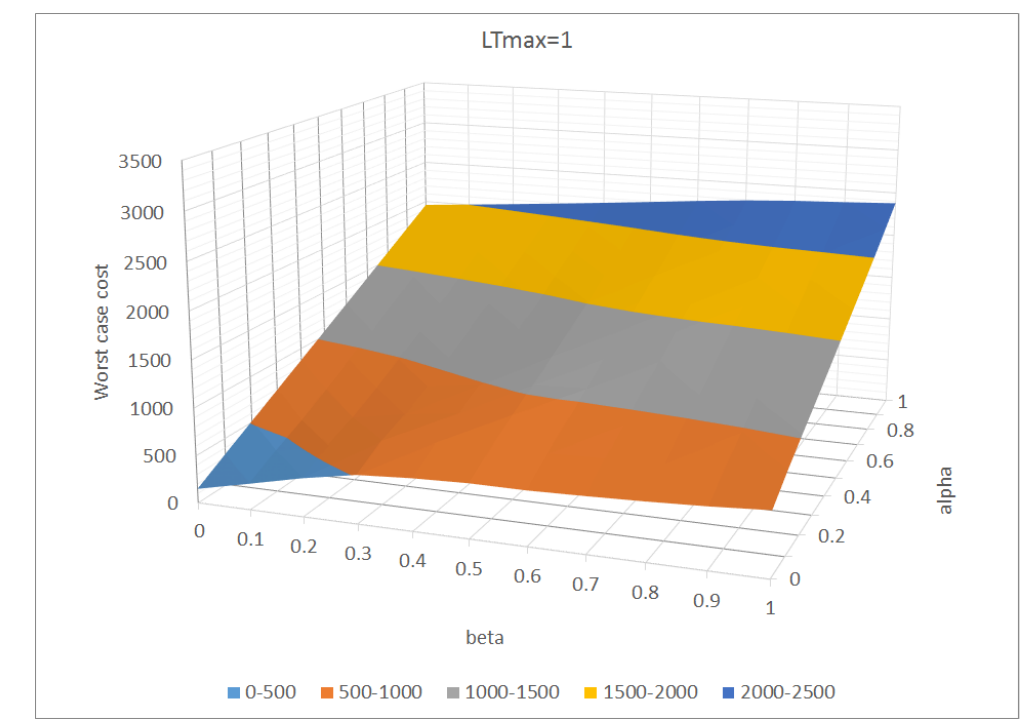

Fig. 3 Surface plot where budget for demand and lead time uncertainty budgets are varied

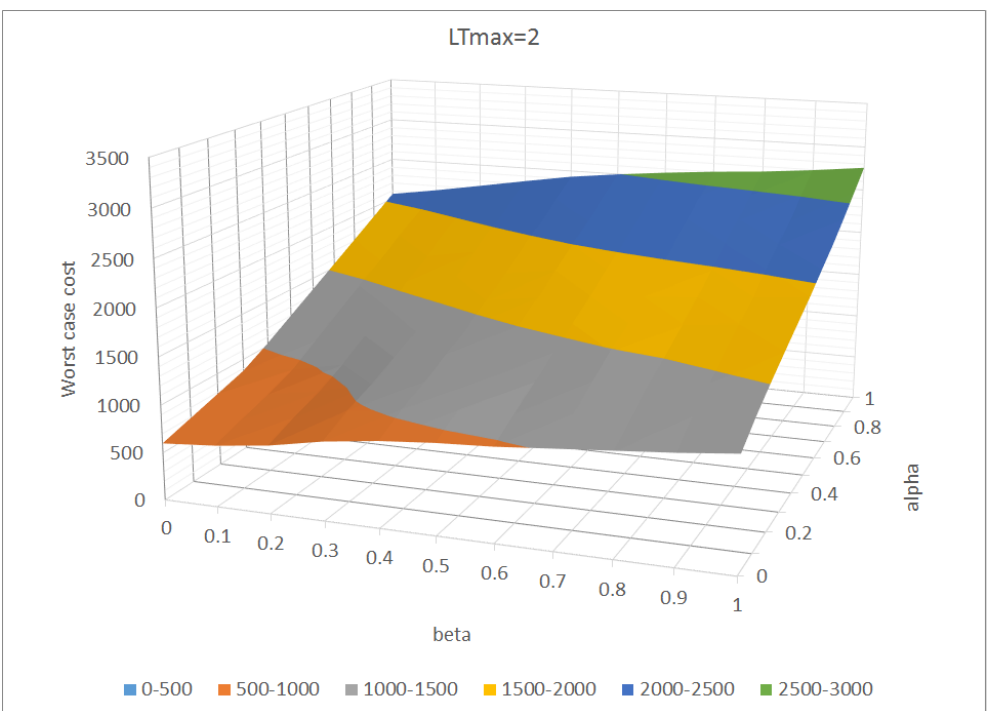

Fig. 4 Surface plot where budget for demand and lead time uncertainty budgets are varied

value. We compare our robust optimization-based approach to the SAA approach. In the SAA approach, we select $N$ samples $\left(\xi_{1}, \ldots, \xi_{N}\right)$, where $\xi_{n}$ represents the $n^{\text {th }}$ realization of lead times $\left(\delta_{i j}^{n}\right.$, where $\left.i=1, \ldots, T, j=i, \ldots, T\right)$ and demands $\left(d_{i}^{n}\right.$, where $\left.i=1, \ldots, T\right)$ for the full time horizon. The following is the SAA formulation for the static policy: 


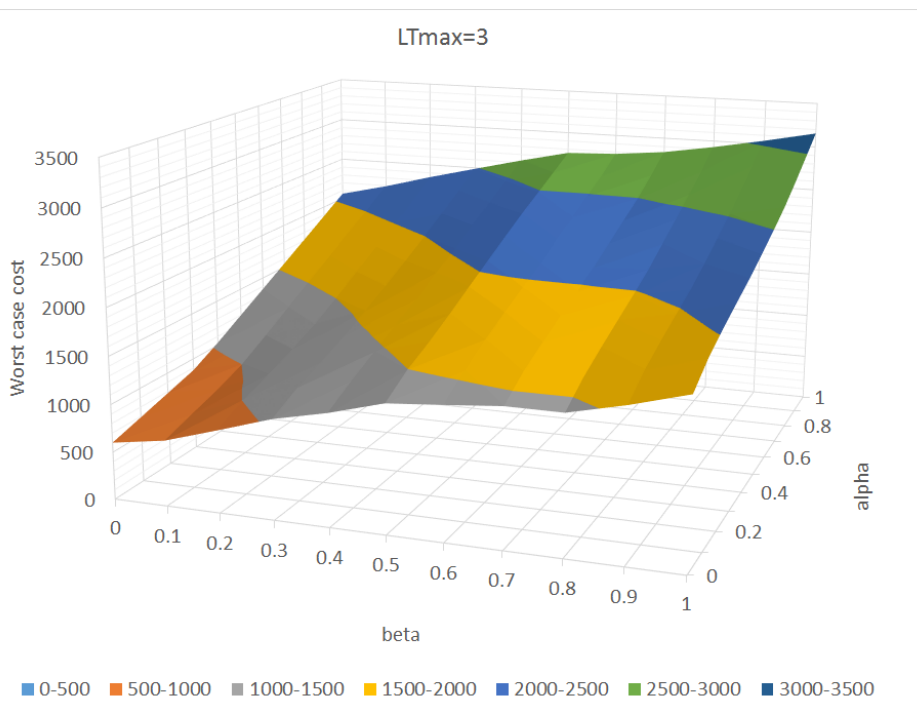

Fig. 5 Surface plot where budget for demand and lead time uncertainty budgets are varied

Table 6 Worst-case costs for specific instances from the example used for Figures 3, 4, and 5 where $\alpha$ (resp. $\beta$ ) are fixed at 0.5 and $\beta$ (resp. $\alpha$ ) are varied from 0 to 1

\begin{tabular}{|c|c|c|c|c|c|c|}
\hline & \multicolumn{3}{|c|}{ Worst Case Costs } & \multicolumn{3}{|c|}{ Worst Case Costs } \\
\hline$L T_{\max }$ & $\begin{array}{c}\text { Model } 1 \\
\alpha=0.5 \\
\beta=0\end{array}$ & $\begin{array}{c}\text { Model } 2 \\
\alpha=0.5, \\
\beta=1\end{array}$ & $\begin{array}{c}\text { \% increase from } \\
\text { Model } 1 \text { to } 2\end{array}$ & $\begin{array}{c}\text { Model } 3 \\
\alpha=0, \\
\beta=0.5\end{array}$ & $\begin{array}{c}\text { Model } 4 \\
\alpha=1 \\
\beta=0.5\end{array}$ & $\begin{array}{l}\text { \% increase from } \\
\text { Model } 3 \text { to } 4\end{array}$ \\
\hline 1 & 1050.4 & 1511.0 & 43.8 & 566.6 & 2194.5 & 287.3 \\
\hline 2 & 1201.2 & 1924.4 & 60.2 & 940.7 & 2495.9 & 165.3 \\
\hline 3 & 1201.2 & 2366.9 & 97.0 & 1326.5 & 2755.0 & 107.7 \\
\hline
\end{tabular}

$$
\begin{aligned}
& \operatorname{minimize} \frac{1}{N}\left(\sum_{n=1}^{N} \sum_{i=1}^{T}\left(c u_{i}+y_{i, n}\right)\right) \\
& y_{i, n} \geq h\left(x_{0}+\sum_{i=1}^{t}\left(\delta_{i t}^{n} u_{i}-d_{i}^{n}\right)\right) \quad i=1, \ldots, T, n=1, \ldots, N \\
& y_{i, n} \geq-b\left(x_{0}+\sum_{i=1}^{t}\left(\delta_{i t}^{n} u_{i}-d_{i}^{n}\right)\right) \quad i=1, \ldots, T, n=1, \ldots, N \\
& u_{i} \geq 0 \quad i=1, \ldots, T .
\end{aligned}
$$

where $N$ is the number of samples. We implement SAA in the following way. For our comparison, we draw 500 samples of demands and lead times for the full time horizon where the demands belong to a uniform distribution between 20 and 30 , and the lead times belong to a discrete uniform distribution between 0 and 3. Then we take the ordering decisions from the SAA problem, and run a Monte Carlo simulation using 100 new samples, using several distributions. The demand 
distributions used are Uniform $(20,30)$ and $\operatorname{Beta}(5,1)$ with a lower bound of 20 and an upper bound of 30, and the lead time distributions used are Uniform $(0,3)$, Two-point (where $\mathrm{P}(\mathrm{LT}=0)=0.25$, and $\mathrm{P}(\mathrm{LT}=3)=0.75$ ), and Triangular (where $\mathrm{P}(\mathrm{LT}=0)=\mathrm{P}(\mathrm{LT}=3)=0.1, \mathrm{P}(\mathrm{LT}=1)=\mathrm{P}(\mathrm{LT}=2)=0.4)$. Tables 7,8 , and 9 show the results from the Monte Carlo simulation for an example using the following parameters: $h=5, b=10, c=1$, and $T=10$.

Since the robust optimization problem is solved for the full range of $0 \leq \alpha \leq 1$ and $0 \leq \beta \leq 1$ in increments of 0.1 , we have $11 * 11=121$ solutions to each problem. In the following tables, we report the lowest, median, and highest values to have a comparison to the SAA problem. The numbers highlighted in bold in Tables 7,8 , and 9 are the objective values of the robust solutions that are smaller than the SAA results.

Table 7 Average Cost from Monte Carlo simulation (100 replications) for Robust Optimization Problem and SAA Problem where demands and lead times are drawn from uniform distributions. Numbers highlighted in bold are the objective values of the robust solutions that are smaller than the SAA results

\begin{tabular}{|c|c|c|c|c|c|c|}
\hline Lead Time Distribution & \multicolumn{2}{|c|}{ Uniform } & \multicolumn{2}{c|}{ 2-point } & \multicolumn{2}{c|}{ Triangular } \\
\hline Demand Distr & Uni & Beta & Uni & Beta & Uni & Beta \\
\hline SAA & 1142 & 2258 & 1420 & 2896 & 1146 & 2384 \\
Robust(lowest) & 1178 & $\mathbf{1 8 4 2}$ & $\mathbf{9 9 0}$ & $\mathbf{1 5 6 1}$ & $\mathbf{1 1 1 8}$ & $\mathbf{1 7 9 9}$ \\
Robust(median) & 1373 & $\mathbf{2 1 0 1}$ & $\mathbf{1 4 1 2}$ & $\mathbf{1 9 7 5}$ & 1360 & $\mathbf{2 3 6 7}$ \\
Robust(highest) & 1933 & 2942 & 2068 & $\mathbf{2 3 2 7}$ & 1969 & 3252 \\
\hline
\end{tabular}

Table 8 Maximum Cost from Monte Carlo simulation (100 replications) for Robust Optimization Problem and SAA Problem where demands and lead times are drawn from uniform distributions. Numbers highlighted in bold are the objective values of the robust solutions that are smaller than the SAA results

\begin{tabular}{|c|c|c|c|c|c|c|}
\hline Lead Time Distribution & \multicolumn{2}{|c|}{ Uniform } & \multicolumn{2}{c|}{ 2-point } & \multicolumn{2}{c|}{ Triangular } \\
\hline Demand Distr & Uni & Beta & Uni & Beta & Uni & Beta \\
\hline SAA & 3269 & 3441 & 2963 & 4203 & 2474 & 3236 \\
Robust(lowest) & $\mathbf{1 1 7 8}$ & $\mathbf{2 7 1 5}$ & $\mathbf{1 8 4 4}$ & $\mathbf{2 3 5 7}$ & $\mathbf{2 0 1 0}$ & $\mathbf{2 7 1 6}$ \\
Robust(median) & $\mathbf{1 3 7 3}$ & $\mathbf{3 1 4 7}$ & $\mathbf{2 2 2 0}$ & $\mathbf{3 3 1 7}$ & $\mathbf{2 4 0 2}$ & 3256 \\
Robust(highest) & $\mathbf{1 9 3 3}$ & 4205 & $\mathbf{2 6 0 3}$ & 4305 & 3461 & 4247 \\
\hline
\end{tabular}

Table 7 shows that the RO solution performs well compared to SAA for several ranges of the budget parameters even though SAA explicitly minimizes average cost while the RO objective is to minimize the maximum cost. SAA dominates RO in terms of average cost under the uniform lead time, uniform demand scenario. This is not surprising since these are the distributions from which SAA was sampled. Tables 8 and 9 indicate that RO provides more stable and robust solutions compared to SAA in terms of standard deviation and worst case solution.

Table 10 shows two specific robust optimization solutions compared to SAA. SAA is generated using uniform demand and uniform lead time distributions. $\mathrm{RO}(\alpha=\beta=0.3)$ is a $\mathrm{RO}$ model with low uncertainty budget, while $\operatorname{RO}(\alpha=$ $\beta=0.9)$ is a RO model where a high uncertainty budget is chosen. In the top 
Table 9 Standard Deviation of Cost from Monte Carlo simulation (100 replications) for Robust Optimization Problem and SAA Problem where demands and lead times are drawn from uniform distributions. Numbers highlighted in bold are the objective values of the robust solutions that are smaller than the SAA results

\begin{tabular}{|c|c|c|c|c|c|c|}
\hline Lead Time Distribution & \multicolumn{2}{|c|}{ Uniform } & \multicolumn{2}{c|}{ 2-point } & \multicolumn{2}{c|}{ Triangular } \\
\hline Demand Distr & Uni & Beta & Uni & Beta & Uni & Beta \\
\hline SAA & 364.2 & 550.8 & 248.8 & 627.4 & 315.9 & 420.3 \\
Robust(lowest) & $\mathbf{2 1 5 . 8}$ & $\mathbf{2 4 3 . 1}$ & $\mathbf{1 8 3 . 7}$ & $\mathbf{1 5 5 . 7}$ & $\mathbf{1 8 0 . 9}$ & $\mathbf{2 7 1 . 3}$ \\
Robust(median) & $\mathbf{2 9 6 . 0}$ & $\mathbf{4 3 7 . 4}$ & 257.6 & $\mathbf{3 6 8 . 9}$ & $\mathbf{2 5 8 . 7}$ & $\mathbf{3 5 8 . 7}$ \\
Robust(highest) & 532.2 & 618.1 & 395.3 & 688.4 & 512.4 & 482.1 \\
\hline
\end{tabular}

section of the table, the out-of-sample distribution is the same as the in-sample SAA distribution. We see that $\mathrm{RO}(\alpha=\beta=0.3)$ performs similarly to SAA with slightly increased average cost, and decreased maximum cost and better stability. The average cost of $\mathrm{RO}(\alpha=\beta=0.9)$ is $54.6 \%$ higher than SAA, but the maximum cost is much lower, and the stability is higher.

In the second set of results from Table 10 , the out-of-sample distribution is Beta for demand, and same as in-sample for lead time. Both RO solutions outperform SAA in all three performance measures. In particular, the stability for $\mathrm{RO}(\alpha=\beta=0.9)$ is better.

In the third set of results from Table 10, the out-of-sample distribution is same as in-sample for demand, and Two-Point for lead time. RO $(\alpha=\beta=0.9)$ has better stability than SAA, and lower maximum cost, with the tradeoff of increased average cost (20\% higher). RO $(\alpha=\beta=0.3)$ has higher average cost and maximum cost than SAA. This may be due to the under-estimate for budget.

Finally, when the out-of-sample distribution is Beta for demand, and TwoPoint for lead time, both RO solutions have good performance compared to SAA in this case.

Table 10 Comparison of two RO solutions to SAA

\begin{tabular}{|c|c|c|c|c|}
\hline Out-Of Sample Dist. & Measure & SAA & $\begin{array}{c}\text { RO } \\
(\alpha=\beta=0.3)\end{array}$ & $\begin{array}{c}\text { RO } \\
(\alpha=\beta=0.9)\end{array}$ \\
\hline$d \sim$ Uniform, & Avg Cost & 1142 & $1238(8.4 \%)$ & $1765(54.6 \%)$ \\
LT $\sim$ Uniform & Max Cost & 3269 & $2762(-15.5 \%)$ & $2463(-24.7 \%)$ \\
& Std. Dev & 364.2 & $326.7(-10.3 \%)$ & $257.9(-26.7 \%)$ \\
\hline$d \sim$ Beta, & Avg Cost & 2258 & $2103(-6.9 \%)$ & $2035(-9.9 \%)$ \\
LT $\sim$ Uniform & Max Cost & 3341 & $3172(-5.1 \%)$ & $2821(-15.6 \%)$ \\
& Std. Dev & 550.8 & $512.6(-6.9 \%)$ & $257.0(-53.3 \%)$ \\
\hline$d \sim$ Uniform, & Avg Cost & 1420 & $1572(10.7 \%)$ & $1704(20.0 \%)$ \\
LT $\sim$ Two-Point & Max Cost & 2963 & $3575(20.6 \%)$ & $2407(-18.8 \%)$ \\
& Std. Dev & 469.5 & $441.8(-5.9 \%)$ & $246.5(-52.5 \%)$ \\
\hline$d \sim$ Beta, & Avg Cost & 2896 & $2908(0.4 \%)$ & $2259(-22.0 \%)$ \\
LT $\sim$ Two - Point & Max Cost & 4203 & $4103(-2.3 \%)$ & $2922(-30.5 \%)$ \\
& Std. Dev & 627.4 & $640.8(2.1 \%)$ & $350.8(-44.1 \%)$ \\
\hline
\end{tabular}

In Table 11 we show the ranges of budget parameters $(\alpha$ and $\beta$ ) that allow RO to outperform SAA in the previous Monte Carlo simulation. RO has a better maximum cost compared to SAA on a wide range of budget parameters for all 
demand/lead time distribution combinations. The average cost of RO can outperform SAA on wide range of parameters when the demand is simulated from a Beta distribution, however under uniformly distributed demand the average cost only outperformed SAA over a narrow range of parameters where $\alpha$ and $\beta$ were usually small.

Table 11 Ranges of $\alpha$ and $\beta$ where RO outperforms SAA with respect to average cost and maximum cost from a Monte Carlo simulation (100 replications)

\begin{tabular}{|c|c|c|c|}
\hline $\begin{array}{l}\text { Demand } \\
\text { distr. }\end{array}$ & $\begin{array}{l}\text { Lead time } \\
\text { distr. }\end{array}$ & $\begin{array}{c}\text { Range where RO outperforms } \\
\text { SAA wrt Avg Cost }\end{array}$ & $\begin{array}{l}\text { Range where RO outperforms } \\
\text { SAA wrt Maximum Cost }\end{array}$ \\
\hline \multirow{3}{*}{ Uniform } & Uniform & None & $\begin{array}{l}\alpha \in[0.4,1], \forall \beta \\
\beta \in[0.3,1], \forall \alpha\end{array}$ \\
\hline & Two-point & $\begin{array}{c}\alpha \in[0.2,1] \text { where } \beta \in[0,0.2] ; \\
\alpha \in[0.1,0.4] \text { where } \beta \in[0.2,0.5]\end{array}$ & $113 / 121$ cases outperform SAA \\
\hline & Triangular & $\begin{array}{l}\text { 23/121 cases outperform SAA; } \\
\text { discontinuous range of } \alpha \text { and } \beta\end{array}$ & $\begin{array}{c}\alpha \in[0.6,1], \forall \beta \\
\beta \in[0.3,1], \forall \alpha\end{array}$ \\
\hline \multirow{3}{*}{ Beta } & Uniform & $\begin{array}{c}\alpha \in[0.1,1] \text { where } \beta \in[0.3,1] \\
\alpha \in[0.8,1], \forall \beta\end{array}$ & $\alpha \in[0.1,1]$ where $\beta \in[0.3,1]$ \\
\hline & Two-point & $\forall \alpha, \forall \beta$ & $118 / 121$ cases outperform SAA \\
\hline & Triangular & $\begin{array}{l}\alpha \in[0.7,1], \forall \beta \\
\beta \in[0.4,1], \forall \alpha\end{array}$ & $\begin{array}{l}\alpha \in[0.7,1], \forall \beta \\
\beta \in[0.4,1], \forall \alpha\end{array}$ \\
\hline
\end{tabular}

\subsection{Results for the Central Limit Theorem Based Uncertainty Set}

In this section we investigate, through computational experiments, the benefits of adding to our model uncertainty sets based on the CLT for demand, $U_{\epsilon}^{d}$, and lead times, $U_{\epsilon}^{L T}$ which were previously defined. Specifically, we investigate the reduction of conservatism in the solution that may be attributed to using these uncertainty sets.

In order for the CLT to hold, so that the partial sums are approximately normally distributed, a well-known rule of thumb is the requirement of a sample size of at least 30 . In this section we examine only instances where $T \geq 30$ in order to satisfy this requirement. Furthermore, we focus on basestock policy models in this section for two reasons: First, as shown in Tables 4 and 5, the basestock models tend to be solved much faster than static models. Second, the scalar decision variable (the order-up-to level) that is determined by the basestock policy optimization may be an effective, and simple, comparison tool between instances.

Table 12 shows the effect of tightening CLT uncertainty set parameters on the worst-case cost for robust basestock models where high budget uncertainty is allowed (i.e., $\alpha=\beta=1$ ). Certain extreme realizations cannot occur when the CLT constraint is binding, such as "all orders arriving with zero lead time", or "all orders arriving with maximum lead time."

For the case of $h=b=10$ (top table), the difference in worst-case cost between $\Gamma=0$ and high CLT uncertainty $(\Gamma=100)$ ranges from $6.3 \%$ to $16.4 \%$. Note that there is no clear trend for basestock levels when $\Gamma$ is changed. One reason for this may be that an equal value for holding and backorder costs allows worst-case values to have either high inventory or high backorders. 
Table 12 Objective values (WC is the best worst-case solution) and corresponding basestock levels (BS) for the solutions. Gamma=100 corresponds to relaxing the CLT uncertainty set so it is non-binding. (Note: $\Gamma$ refers to the parameter setting for both $\Gamma_{L T}$ and $\Gamma_{d}$ )

\begin{tabular}{|c|c|c|c|c|c|c|c|c|}
\hline \multicolumn{9}{|c|}{$\mathrm{T}=30, \mathrm{c}=3, \mathrm{~h}=10, \mathrm{~b}=10, \alpha=\beta=1$} \\
\hline \multirow[b]{2}{*}{ Gamma } & \multicolumn{2}{|c|}{$L T_{\max }=2$} & \multicolumn{2}{|c|}{$L T_{\max }=4$} & \multicolumn{2}{|c|}{$L T_{\max }=6$} & \multicolumn{2}{|c|}{$L T_{\max }=8$} \\
\hline & WC & $\mathrm{BS}$ & $\mathrm{WC}$ & $\mathrm{BS}$ & WC & $\mathrm{BS}$ & WC & $\mathrm{BS}$ \\
\hline 100 & 9060 & 36 & 15250 & 57 & 21440 & 77 & 27630 & 97 \\
\hline 4 & 9043 & 31 & 15107 & 46 & 21068 & 61 & 26928 & 76 \\
\hline 3 & 9021 & 29 & 15052 & 44 & 20984 & 59 & 26703 & 37 \\
\hline 2 & 8979 & 28 & 14981 & 43 & 20560 & 31 & 25290 & 43 \\
\hline 1 & 8918 & 20 & 14449 & 26 & 19514 & 34 & 24209 & 44 \\
\hline 0 & 8480 & 20 & 13782 & 32 & 18619 & 41 & 23108 & 52 \\
\hline \multicolumn{9}{|c|}{$\mathrm{T}=30, \mathrm{c}=3, \mathrm{~h}=10, \mathrm{~b}=50, \alpha=\beta=1$} \\
\hline & \multicolumn{2}{|c|}{$L T_{\max }=2$} & \multicolumn{2}{|c|}{$L T_{\max }=4$} & \multicolumn{2}{|c|}{$L T_{\max }=6$} & \multicolumn{2}{|c|}{$L T_{\max }=8$} \\
\hline Gamma & $\mathrm{WC}$ & $\mathrm{BS}$ & $\mathrm{WC}$ & $\mathrm{BS}$ & $\mathrm{WC}$ & BS & $\mathrm{WC}$ & $\mathrm{BS}$ \\
\hline 100 & 15893 & 52 & 30877 & 86 & 48527 & 119 & 68843 & 152 \\
\hline 4 & 15403 & 53 & 30502 & 87 & 48266 & 120 & 68697 & 153 \\
\hline 3 & 15017 & 57 & 30127 & 93 & 48241 & 120 & 68702 & 153 \\
\hline 2 & 14597 & 57 & 29407 & 93 & 47450 & 130 & 68540 & 160 \\
\hline 1 & 14177 & 57 & 28687 & 93 & 46430 & 130 & 67607 & 167 \\
\hline 0 & 13886 & 56 & 27967 & 93 & 45510 & 130 & 66387 & 167 \\
\hline \multicolumn{9}{|c|}{$\mathrm{T}=30, \mathrm{c}=3, \mathrm{~h}=10, \mathrm{~b}=100, \alpha=\beta=1$} \\
\hline & \multicolumn{2}{|c|}{$L T_{\max }=2$} & \multicolumn{2}{|c|}{$L T_{\max }=4$} & \multicolumn{2}{|c|}{$L T_{\max }=6$} & \multicolumn{2}{|c|}{$L T_{\max }=8$} \\
\hline Gamma & $\mathrm{WC}$ & $\mathrm{BS}$ & $\mathrm{WC}$ & $\mathrm{BS}$ & $\mathrm{WC}$ & $\mathrm{BS}$ & WC & $\mathrm{BS}$ \\
\hline 100 & 19901 & 56 & 42610 & 92 & 71864 & 128 & 107755 & 165 \\
\hline 4 & 18966 & 58 & 41644 & 96 & 71479 & 129 & 107472 & 165 \\
\hline 3 & 18446 & 58 & 40924 & 96 & 71479 & 129 & 107472 & 165 \\
\hline 2 & 18026 & 58 & 40204 & 96 & 69555 & 135 & 106179 & 173 \\
\hline 1 & 17606 & 58 & 39484 & 96 & 68535 & 135 & 104958 & 173 \\
\hline 0 & 17185 & 58 & 38764 & 96 & 67615 & 135 & 103738 & 173 \\
\hline
\end{tabular}

For the case of $h=10, b=50$ (middle table), the trend for basestock levels is more clear. As the solution becomes less conservative (i.e., worst-case cost decreases) the basestock level increases. The same trend holds for the bottom table where $h=10, b=100$ (bottom table). The difference in worst-case cost between $\Gamma=0$ and high CLT uncertainty $(\Gamma=100)$ for these two instances ranges from about $3-13 \%$.

\section{Concluding Remarks}

In this paper, several robust optimization models for inventory control under uncertain demand and uncertain lead time are proposed. We describe several approaches for robust optimization, including budget uncertainty sets and CLT-based uncertainty sets.

Our numerical results indicate that standard robust optimization methods using epigraph reformulations are overly conservative for the multi-period inventory control problem under demand and lead time uncertainty due to their inherent overestimation of total cost even when costs and demands are stationary. This is due to the decoupling of the robust optimization subproblems that are solved. We 
present new budget-type lead time uncertainty sets and CLT-type uncertainty sets to handle demand and lead time uncertainty.

We compare our demand and lead time budget RO approach to the samplingbased SAA method. The RO solution performs well compared to SAA for several ranges of the budget parameters even though SAA explicitly minimizes average cost while the RO objective is to minimize the maximum cost. The computational results indicate that $\mathrm{RO}$ provides more stable and robust solutions compared to SAA in terms of standard deviation and worst case solutions, especially when the realized distribution is different than the sampled distribution.

The research presented in this paper can be considered the start of a framework for modeling lead time uncertainty using robust optimization. Future work can involve extending this approach to a more general setting: for example including fixed costs, and considering multi-echelon supply chains. Using this approach, it would be interesting to consider adaptive policies that explicitly consider the number and age of outstanding orders in the ordering decisions.

\section{References}

Alvarez, P. P. and Vera, J. R. (2011). Application of robust optimization to the sawmill planning problem. Annals of Operations Research, pages 1-19.

Ang, M., Lim, Y. F., and Sim, M. (2012). Robust storage assignment in unit-load warehouses. Management Science, 58(11):2114-2130.

Aouam, T. and Brahimi, N. (2013). Integrated production planning and order acceptance under uncertainty: A robust optimization approach. European Journal of Operational Research, 228(3):501-515.

Bandi, C. and Berstimas, D. (2012). Tractable stochastic analysis in high dimensions via robust optimization. Mathematical Programming, 134(1):23-70.

Beck, A. and Ben-Tal, A. (2009). Duality in robust optimization: Primal worst equals dual best. Operations Research Letters, 37(1):1-6.

Ben-Tal, A., El Ghaoui, L., and Nemirovski, A. (2009). Robust optimization. Princeton University Press.

Ben-Tal, A., Golany, B., Nemirovski, A., and Vial, J. (2005). Retailer-supplier flexible commitments contracts: a robust optimization approach. Manufacturing and Service Operations Management, 7(3):248-271.

Ben-Tal, A., Goryashko, A., Guslitzer, E., and Nemirovski, A. (2004). Adjustable robust solutions of uncertain linear programs. Mathematical Programming, 99(2):351-376.

Ben-Tal, A. and Nemirovski, A. (1999). Robust solutions of uncertain linear programs. Operations Research Letters, 25(1):1-13.

Bertsimas, D. and Sim, M. (2004). The price of robustness. Operations Research, $52(1): 35-53$.

Bertsimas, D. and Thiele, A. (2006). A robust optimization approach to inventory theory. Operations Research, 54(1):150-168.

Bienstock, D. and Ozbay, N. (2008). Computing robust basestock levels. Discrete Optimization, 5(2):389-414.

Bohle, C., Maturana, S., and Vera, J. (2010). A robust optimization approach to wine grape harvesting scheduling. European Journal of Operational Research, 200(1):245-252. 
Carlsson, D., Flisberg, P., and Rönnqvist, M. (2014). Using robust optimization for distribution and inventory planning for a large pulp producer. Computers \& Operations Research, 44:214-225.

Clark, A. and Scarf, H. (1960). Optimal policies for a multi-echelon inventory problem. Management Science, 6(4):475-490.

Denton, B. T., Miller, A. J., Balasubramanian, H. J., and Huschka, T. R. (2010). Optimal allocation of surgery blocks to operating rooms under uncertainty. $O p$ erations Research, 58(4):802-816.

Gabrel, V., Murat, C., and Thiele, A. (2014). Recent advances in robust optimization: An overview. European Journal of Operational Research, 235(3):471-483.

Gorissen, B. L. and Den Hertog, D. (2013). Robust counterparts of inequalities containing sums of maxima of linear functions. European Journal of Operational Research, 227(1):30-43.

Hayya, J., Bagchi, U., Kim, J., and Sun, D. (2008). On static stochastic order crossover. International Journal of Production Economics, 114:404-413.

He, X., Xu, S., and Ord, J. (1998). An inventory model with order crossover. Operations Research, 46(3):S112-S119.

José Alem, D. and Morabito, R. (2012). Production planning in furniture settings via robust optimization. Computers \&f Operations Research, 39(2):139-150.

Minoux, M. (2009). Robust linear programming with right-hand-side uncertainty, duality, and applications. In Floudas, L. and Pardolas, P., editors, Encyclopedia of Optimization, 2nd edn. Springer, Boston, US.

Minoux, M. (2011). On two-stage robust LP with RHS uncertainty: complexity results and applications. Journal of Global Optimization, 49(3):521-537.

Movahed, K. K. and Zhang, Z.-H. (2013). Robust design of (s, s) inventory policy parameters in supply chains with demand and lead time uncertainties. International Journal of Systems Science, (ahead-of-print):1-11.

Mulvey, J. M., Vanderbei, R. J., and Zenios, S. A. (1995). Robust optimization of large-scale systems. Operations Research, 43(2):264-281.

Riezebos, J. (2006). Inventory order crossovers. International Journal of Production Economics, 104(2):666-675.

Rikun, A. (2011). Applications of Robust Optimization to Queueing and Inventory Systems. PhD thesis, Massachusetts Institute of Technology.

Soyster, A. (1973). Convex programming with set-inclusive constraints and applications to inexact linear programming. Operations Research, 21(5):1154-1157.

Soyster, A. and Murphy, F. (2013). A unifying framework for duality and modeling in robust linear programs. Omega, 41(6):984-997.

Varas, M., Maturana, S., Pascual, R., Vargas, I., and Vera, J. (2014). Scheduling production for a sawmill: A robust optimization approach. International Journal of Production Economics, 150:37-51.

Wei, C., Li, Y., and Cai, X. (2011). Robust optimal policies of production and inventory with uncertain returns and demand. International Journal of Production Economics, 134(2):357-367.

Zhang, M. (2011). Two-stage minimax regret robust uncapacitated lot-sizing problems with demand uncertainty. Operations Research Letters, 39(5):342-345.

Zipkin, P. (2000). Foundations of Inventory Management. McGraw-Hill. 